\title{
The Cuntz semigroup as an invariant for $\mathrm{C}^{*}$-algebras
}

\author{
Kristofer T. Coward, George A. Elliott, and Cristian Ivanescu
}

\begin{abstract}
A category is described to which the Cuntz semigroup belongs and as a functor into which it preserves inductive limits.
\end{abstract}

1. Recently, Toms in [26] used the refinement of the invariant $\mathrm{K}_{0}$ introduced by Cuntz almost thirty years ago in [4] to show that certain $\mathrm{C}^{*}$-algebras are not isomorphic. Anticipating the possible use of this invariant to establish isomorphism, we take the liberty of reporting some observations concerning it. (In particular, we present what might be viewed as an embryonic isomorphism theorem.)

One of the first things that might be noted in connection with this invariant, which considers, instead of the finitely generated projective modules over a given $\mathrm{C}^{*}$-algebra, the larger class of modules consisting of the countably generated Hilbert $\mathrm{C}^{*}$-modules (see [12], [15], and [21]; see also [10]) is that, whereas the equivalence relation between finitely generated projective modules would appear to be inevitable, namely, just isomorphism, in the wider setting of Hilbert $\mathrm{C}^{*}$-modules it is no longer quite so clear what the equivalence relation should be. While it is tempting just to choose isomorphism again, one should note that, even in the stably finite case (which is perhaps the case that this invariant is of most interest), whereas the isomorphism classes of algebraically finitely generated Hilbert $\mathrm{C}^{*}$-modules (which are of course also algebraically projective, and up to isomorphism exhaust the finitely generated projective modules) form an ordered set with respect to inclusion (in other words, if each of two such modules is isomorphic to a submodule of the other, then they must be isomorphic - indeed, any two such isomorphisms, from each of

The research of the second and third authors was supported by grants from the Natural Sciences and Engineering Research Council of Canada.

AMS 2000 Mathematics Subject Classification. Primary: 46L05, 46L35, 46M15; Secondary: 19K14. 
two such modules onto a submodule of the other, must, by stable finiteness, already be surjective - and so constitute an isomorphism of the two given modules), this would seem to be almost completely open for isomorphism classes of Hilbert $\mathrm{C}^{*}$ modules. (Note that we are referring here to the stably finite case; interestingly, in the purely infinite simple case, the countably generated Hilbert $\mathrm{C}^{*}$-modules which are not finitely generated as modules are all isomorphic — see 4.1 .3 of [24] — and in particular the inclusion relation for isomorphism classes is antisymmetric! In the case of real rank zero and stable rank one, which is a very small subset of the stably finite case, the proof that the Murray-von Neumann equivalence class of a multiplier projection of an AF algebra is determined by the set of equivalence classes of projections in the $\mathrm{C}^{*}$-algebra that it majorizes - see [5] - which uses cancellation, known (see 6.5.1 and 6.5.2 of [1]) to be equivalent to stable rank one in the presence of real rank zero - shows that the isomorphism classes of countably generated Hilbert $\mathrm{C}^{*}$-modules are determined by the isomorphism classes of the algebraically finitely generated ones they include, and it follows again immediately that the inclusion relation on isomorphism classes is antisymmetric.) (In Theorem 3 below we shall show that this holds without assuming real rank zero-provided that stable rank one is still assumed.) (Note that antisymmetry fails for inclusion of isomorphism classes of finitely generated projective modules in the Cuntz algebra $\mathcal{O}_{n}$ for $n \geq 3$.) Nevertheless, one would obtain an ordered semigroup by just antisymmetrizing the inclusion relation on isomorphism classes.

Cuntz's choice of equivalence relation is a weaker one again, of an approximative nature (although there still seems to be no indication whether it is different from the one just described - i.e., two-way inclusion of isomorphism classes - or, in the stably finite case, from just isomorphism for that matter). It should be emphasized that the present formulation of Cuntz's invariant, in terms of (countably generated) Hilbert $\mathrm{C}^{*}$-modules (it could be done alternatively-but slightly less convenientlyin particular one would need Brown's theorem to construct suprema! - in terms of (singly generated) hereditary sub-C*-algebras of the stabilization of the $\mathrm{C}^{*}$-algebra) is important for our determination of the abstract structure of this invariant (see below). Accordingly, let us describe Cuntz's equivalence relation in the setting of Hilbert $\mathrm{C}^{*}$-modules.

Cuntz's equivalence relation, like the one considered above, is based on a preorder relation, compatible with direct sum of Hilbert $\mathrm{C}^{*}$-modules and so again giving rise to an ordered abelian semigroup (possibly the same one!). For our purposes, it seems most appropriate to describe it in terms of the notion of compact inclusion: let us say that a closed submodule of a given Hilbert $\mathrm{C}^{*}$-module 
over a given $\mathrm{C}^{*}$-algebra - let us call this a subobject - is compactly contained as a subobject - and denote this relation by $\subset \subset$-if there is a compact self-adjoint endomorphism of the larger Hilbert $\mathrm{C}^{*}$-module (see [15]) which is equal to the identity on the smaller one.

Given two Hilbert $\mathrm{C}^{*}$-modules over a given $\mathrm{C}^{*}$-algebra, let us say that they are equivalent in the sense of Cuntz if, up to isomorphism, they have the same compactly contained subobjects. In other words a third Hilbert $\mathrm{C}^{*}$-module should be isomorphic to a compactly contained subobject of one of these two Hilbert $\mathrm{C}^{*}$ modules if and only if it is isomorphic to a compactly contained subobject of the other. Clearly this is an equivalence relation, and it can be extended to a pre-order relation by requiring every compactly contained subobject of the first of two given Hilbert $\mathrm{C}^{*}$-modules to be isomorphic to a compactly contained subobject of the second. Let us pass to the space of Cuntz equivalence classes, so that the pre-order becomes an order, and let us denote the resulting order relation by $\leq$.

The first comment that should be made concerning the relation $\leq$ on Cuntz equivalence classes is that while it is trivially an order relation (as it just refers to comparision by inclusion of the sets of isomorphism classes of compactly contained subobjects of two given objects), it is not quite obvious that this relation even holds - as it of course should! - when one object is contained in another as a subobject. Let us verify this. If $X$ and $Y$ are countably generated Hilbert $\mathrm{C}^{*}$ modules with $X \subseteq Y$, and if $X_{0}$ is a compactly contained subobject of $X$-i.e., if $X_{0} \subset \subset X-$, in other words, if $X_{0} \subseteq X$ and some compact self-adjoint endomorphism of $X$ is the identity on $X_{0}$, then this compact endomorphism extends in a natural way to a compact endomorphism of $Y \supseteq X$ (as follows from Theorem 2 of [14], which shows that the obvious extension of a finite-rank endomorphism has the same norm), which is still the identity on $X_{0}$, and still self-adjoint, and so $X_{0} \subset \subset Y$; this proves that $[X] \leq[Y]$, where $[X]$ and $[Y]$ denote the Cuntz equivalence classes of $X$ and $Y$.

The second comment that should be made concerning the relation $\leq$ between equivalence classes is that it is compatible with addition, but that, even in the special case of equality, this must be proved. In other words, even the fact that the Cuntz semigroup exists, in the present (nonapproximative) context of countably generated Hilbert $\mathrm{C}^{*}$-modules, isomorphism, and compact containment, must form part of our analysis of this invariant. (Of course, one approach would be just to reconcile the present equivalence relation with the approximative one of Cuntzwhich, as is easily seen, does amount to a relation on (countably generated) Hilbert $\mathrm{C}^{*}$-modules, weaker in general than isomorphism. Cuntz, as it happens, only looked 
at finitely generated Hilbert $\mathrm{C}^{*}$-modules, rather than arbitrary countably generated Hilbert $\mathrm{C}^{*}$-modules, but this is immaterial. Indeed, in the case of a stable $\mathrm{C}^{*}$ algebra, every countably generated Hilbert $\mathrm{C}^{*}$-modules is singly generated, and in fact is a closed right ideal, i.e., a subobject of the $\mathrm{C}^{*}$-algebra considered as a Hilbert $\mathrm{C}^{*}$-module. In the general case one has to look at (countably generated) subobjects of the countably infinite direct sum of copies of the given $\mathrm{C}^{*}$-algebra. Note that only when the given $\mathrm{C}^{*}$-algebra has a countable approximate unit is the Hilbert $\mathrm{C}^{*}$-module arising from it countably generated. That Cuntz's equivalence relation viewed as a relation on (countably generated) Hilbert $\mathrm{C}^{*}$-modules coincides with ours - see Appendix 6-follows from a functional calculus lemma of Kirchberg and Rørdam-Lemma 2.2 of [17] — which is in fact the main technical tool in our analysis below.)

Theorem. The Cuntz invariant (as defined above) is an ordered semigroup with zero (the order relation compatible with addition in the sense that if $\left[X_{1}\right] \leq\left[Y_{1}\right]$ and $\left[X_{2}\right] \leq\left[Y_{2}\right]$ then $\left[X_{1} \oplus X_{2}\right] \leq\left[Y_{1} \oplus Y_{2}\right]$, and, also, zero is the smallest element). The order relation has the following two purely order-theoretic properties:

(i) every increasing sequence-equivalently, every countable upward directed sethas a supremum;

(ii) for any element the set of elements compactly contained in it in the ordertheoretic sense-where we say that $x$ is compactly contained in $y$ in the ordertheoretic sense-let us denote this relation by $x<<y$-if, whenever $y_{1} \leq y_{2} \leq \cdots$ is an increasing sequence with supremum greater than or equal to y, eventually $x \leq y_{n}$-is upward directed - also with respect to the stronger relation $<<$ (which is transitive, and even antisymmetric, but not in general reflexive)-and contains an increasing sequence-which may be chosen to be rapidly increasing, i.e. with each term compactly contained in the next-with supremum the given element.

The operation of passing to the supremum of a countable upward directed set and the relation $<<$ of compact containment in the order-theoretic sense are compatible with addition, in the sense that the supremum of the sum $S_{1}+S_{2}$ of two countable upward directed subsets $S_{1}$ and $S_{2}$ (which is of course also upward directed, and so by (i) has a supremum) is the sum of the suprema (in other words $\sup \left(S_{1}+S_{2}\right)=$ $\left.\sup S_{1}+\sup S_{2}\right)$, and the relations $x_{1}<<y_{1}$ and $x_{2}<<y_{2}$ imply $x_{1}+x_{2}<<y_{1}+y_{2}$.

Proof. We must first show, in order to obtain the Cuntz invariant as an ordered semigroup in our approach (even just as a semigroup), that the pre-order relation defined above on countably generated Hilbert $\mathrm{C}^{*}$-modules (over a given $\mathrm{C}^{*}$-algebra) is compatible with addition. Let $X_{1}, X_{2}, Y_{1}$, and $Y_{2}$ be countably generated Hilbert 
$\mathrm{C}^{*}$-modules, suppose that $\left[X_{1}\right] \leq\left[Y_{1}\right]$ and $\left[X_{2}\right] \leq\left[Y_{2}\right]$, i.e., that every compactly contained subobject of $X_{1}$ (where by subobject we mean countably generated closed submodule, considered with the inherited algebra-valued inner product) is isomorphic to a compactly contained subobject of $Y_{1}$ and similarly for $X_{2}$ and $Y_{2}$, and let us show that $\left[X_{1} \oplus X_{2}\right] \leq\left[Y_{1} \oplus Y_{2}\right]$. Let $X$ be a compactly contained subobject of $X_{1} \oplus X_{2}$-i.e., $X \subset \subset X_{1} \oplus X_{2}$-and let us show that $X$ is isomorphic to $Y \subset \subset Y_{1} \oplus Y_{2}$.

Consider first the case that $X=X_{1}^{\prime} \oplus X_{2}^{\prime}$ with $X_{1}^{\prime} \subset \subset X_{1}$ and $X_{2}^{\prime} \subset \subset X_{2}$. In this case, by hypothesis, $X_{1}^{\prime}$ and $X_{2}^{\prime}$ are isomorphic respectively to objects $Y_{1}^{\prime} \subset \subset Y_{1}$ and $Y_{2}^{\prime} \subset \subset Y_{2}$, and hence $X_{1}^{\prime} \oplus X_{2}^{\prime}$ is isomorphic to the subobject $Y_{1}^{\prime} \oplus Y_{2}^{\prime}$ of $Y_{1} \oplus Y_{2}$, which is clearly compactly contained in $Y_{1} \oplus Y_{2}$. (If $b_{1}$ and $b_{2}$ are compact self-adjoint endomorphisms of $Y_{1}$ and $Y_{2}$ acting as the identity on $Y_{1}^{\prime}$ and $Y_{2}^{\prime}$, respectively, then $b_{1} \oplus b_{2}$ is a compact self-adjoint endomorphism of $Y_{1} \oplus Y_{2}$ acting as the identity on $Y_{1}^{\prime} \oplus Y_{2}^{\prime}$.)

The case that $X$ is isomorphic to a subobject $X_{1}^{\prime} \oplus X_{2}^{\prime}$ of $X_{1} \oplus X_{2}$ as above (i.e., with $X_{1}^{\prime} \subset \subset X_{1}$ and $X_{2}^{\prime} \subset \subset X_{2}$ ) of course reduces to the preceding case (that $\left.X=X_{1}^{\prime} \oplus X_{2}^{\prime}\right)$. The case that $X$ is isomorphic to a subobject of such a subobject follows immediately. We shall show below, after first establishing the assertions (i) and (ii) of the theorem without making any reference to addition whatsoever, that this last case is in fact the general case. In anticipation of this, and in deference to the fact that the Cuntz semigroup exists in the literature already - with a different definition that will later be seen to be the same-let us in the meantime anyway refer to the ordered set of Cuntz equivalence classes as the Cuntz semigroup.

Let us proceed to the proof of the first purely order-theoretic assertion of the theorem (the assertion (i)). Let $x_{1} \leq x_{2} \leq \ldots$ be an increasing sequence in the Cuntz semigroup of a given $\mathrm{C}^{*}$-algebra $A$, and let us show that the supremum of the set $\left\{x_{n} ; n=1,2, \cdots\right\}$ exists in this ordered set. The proof consists of two steps, of which the first can be dealt with immediately, whereas the second requires first establishing the second assertion of the theorem.

The first step is to consider the case that the sequence is rapidly increasing, i.e., that $x_{1}<<x_{2}<<\cdots$. In fact, this is disingenuous, and before continuing with the proof, we must introduce a concrete analogue of the relation $<<$, arising from the relation $\subset \subset$ of compact inclusion of a subobject of a Hilbert $\mathrm{C}^{*}$-module. Namely, let us say that $x_{1}$ is compactly contained in $x_{2}$ in the concrete sense, and write $x_{1} \subset \subset x_{2}$-we shall eventually show that this relation is equivalent to $x_{1}<<x_{2}$ !if $x_{1} \leq\left[X_{2}^{\prime}\right]$ for some object (i.e., countably generated Hilbert $\mathrm{C}^{*}$-module) $X_{2}^{\prime}$ such that $X_{2}^{\prime} \subset \subset X_{2}$ for some object $X_{2}$ representing $x_{2}$, i.e., with $\left[X_{2}\right]=x_{2}$. (We 
do not require that $x_{1}=\left[X_{2}^{\prime}\right]$ for some object $X_{2}^{\prime}$ as above, i.e., $X_{2}^{\prime} \subset \subset X_{2}$, with $\left[X_{2}\right]=x_{2}$, although it might be the case that $X_{2}^{\prime}$ can always be chosen in this way.)

More precisely, then, the first step is to consider the case that the sequence is rapidly increasing - written $x_{1} \subset \subset x_{2} \subset \subset \cdots$-in the concrete sense, i.e., in the sense that there exist countably generated Hilbert $A$-modules $X_{1}, X_{2}, X_{2}^{\prime}, X_{3}, X_{3}^{\prime}, \cdots$ with $X_{2}^{\prime} \subset \subset X_{2}, X_{3}^{\prime} \subset \subset X_{3}, \cdots$ such that $\left[X_{n}\right]=x_{n}$ for $n=1,2, \cdots$, and $x_{1} \leq\left[X_{2}^{\prime}\right], x_{2} \leq\left[X_{3}^{\prime}\right], \cdots$ where, as above, $[X]$ denotes the Cuntz equivalence class of $X$. Recall that, as proved above, $X \subset \subset Y$ implies $[X] \leq[Y]$, and so this is indeed a special case $\left(x_{1} \subset \subset x_{2} \subset \subset \cdots\right.$ does imply $\left.x_{1} \leq x_{2} \leq \cdots\right)$.

By the definition of Cuntz equivalence, from $X_{2}^{\prime} \subset \subset X_{2}$ and $\left[X_{2}\right] \leq\left[X_{3}^{\prime}\right]$ it follows that $X_{2}^{\prime}$ is isomorphic to a compactly contained subobject of $X_{3}^{\prime}$. Similarly, $X_{3}^{\prime}$ is isomorphic to a compactly contained subobject of $X_{4}^{\prime}$, and so on. Thus, we have a sequence of $A$-module maps, preserving the $A$-valued inner product and in particular isometric,

$$
X_{2}^{\prime} \rightarrow X_{3}^{\prime} \rightarrow \cdots
$$

for which the image of each object is a compactly contained subobject of the next, and we assert that the inductive limit Hilbert $A$-module $X=\lim _{\rightarrow} X_{n}^{\prime}$ gives rise to the supremum of the classes $x_{1}=\left[X_{1}\right], x_{2}=\left[X_{2}\right], \cdots$ in the Cuntz semigroup of $A$-which are, after all, intertwined with the classes $\left[X_{1}^{\prime}\right],\left[X_{2}^{\prime}\right], \cdots$. In fact, we shall not need to use that the image of $X_{n}^{\prime}$ is a compactly contained subobject of $X_{n+1}^{\prime}$, but just a subobject for each $n=2,3, \cdots$.

In other words, changing notation, we must show that if $X=\lim _{\rightarrow} X_{n}$ for a sequence $X_{1} \rightarrow X_{2} \rightarrow$ of $A$-module maps preserving the $A$-valued inner product, then $[X]=\sup \left[X_{n}\right]$. (As mentioned implicitly this is what is pertinent above, since, in the earlier notation, $\left[X_{1}\right] \leq\left[X_{2}^{\prime}\right] \leq\left[X_{2}\right] \leq\left[X_{3}^{\prime}\right] \leq\left[X_{3}\right] \leq \cdots$.)

To show that the class of $X$ is the supremum of $\left[X_{1}\right],\left[X_{2}\right], \cdots$, we must show that if $Y$ is a countably generated Hilbert $A$-module such that $\left[X_{n}\right] \leq[Y]$ for all $n=1,2, \cdots$ then also $[X] \leq[Y]$. Let $Y$ then be such that $\left[X_{n}\right] \leq[Y]$ for all $n$ in the Cuntz semigroup of $A$, and let $Z$ be a countably generated Hilbert $A$-module such that $Z \subset \subset X$; we must show that $Z \cong Z^{\prime}$ for some subobject $Z^{\prime} \subset \subset Y$. We shall show that in fact $Z \cong Z^{\prime \prime}$ for some $Z^{\prime \prime} \subset \subset X_{n}$ for some $n$. (It follows from this by definition of the inequality $\left[X_{n}\right] \leq[Y]$ that $Z^{\prime \prime} \cong Z^{\prime}$ with $Z^{\prime} \subset \subset Y$ as required.)

Let us show then that $Z \cong Z^{\prime \prime}$ for some $Z^{\prime \prime} \subset \subset X_{n}$. Using the hypothesis $Z \subset \subset X$, choose a compact self-adjoint endomorphism $b$ of $X$ such that $b$ is equal to the identity on $Z \subset \subset X$. We may replace $b$ by $b^{2}$ to ensure that $b$ is positive, and then replace $b$ by a function of $b$ so that, for some $\epsilon>0$, also $(b-\epsilon)_{+}$is the 
identity on $Z$. Again replacing $b$ by a function of $b$ (in the $\mathrm{C}^{*}$-algebra generated by $b$, and so still a compact endomorphism of $X$ ), we may suppose that also yet another positive function of $b$, say $c$, is the identity on $b$ (and still belongs to the algebra of compact endomorphisms of $\mathrm{X}$ ). Choose a sequence $\left(c_{n}\right)$ of positive compact endomorphisms of $X$, with $c_{n}$ arising from a compact endomorphism of $X_{n} \subseteq X$, such that $c_{n}$ converges to $c$. Then $c_{n} b c_{n}$ converges to $c b c=b$, and so, with $\epsilon>0$ as above, by Lemma 2.2 of [17], if $n$ is large enough that $\left\|c_{n} b c_{n}-b\right\|<\epsilon$, then for some compact endomorphism $d_{n}$ of $X$ (which could be chosen to have norm at most one), $d_{n} c_{n} b c_{n} d_{n}^{*}=(b-\epsilon)_{+}$. Note that $c_{n} b c_{n}$ also arises from a compact endomorphism of $X_{n}$ (as these constitute a hereditary sub-C*-algebra of the compact endomorphisms of $X$ ), and so also its positive square root, $g_{n}$, does. Since

$$
\left(d_{n} g_{n}\right)\left(d_{n} g_{n}\right)^{*}=(b-\epsilon)_{+},
$$

the partially isometric part of $d_{n} g_{n}$ in the bidual of the $\mathrm{C}^{*}$-algebra of compact endomorphisms of $X$ therefore determines an isomorphism between a subobject of $X_{n}$ and the subobject of $X$ generated by $(b-\epsilon)_{+} X$ (i.e., the closure of this submodule of $X$-recall that $A$ acts on $X$ on the right). Since $(b-\epsilon)_{+}$acts as the identity on $Z$, the object $Z$ is a subobject of the subobject of $X$ in question, the closure of the range of $(b-\epsilon)_{+}$, and is therefore isomorphic, as desired, to a subobject of (the above subobject of) $X_{n}$.

This completes the first step of the proof of the assertion (i), namely, the consideration of the special case of a rapidly increasing sequence $x_{1} \subset \subset x_{2} \subset \subset \cdots$ in the concrete sense - cf. above - namely - as it turns out, but this step was slightly subtle-, that $x_{n}=\left[X_{n}\right]$ with $X_{1} \subset \subset X_{2} \subset \subset \cdots$. As mentioned above, before proceeding to the second step (the general case!) we must first establish the second assertion of the theorem. Actually, this is not quite correct; it will suffice to establish the assertion (ii) with the purely order-theoretic relation $x<<y$ replaced by the concrete relation $x \subset \subset y$ introduced above $\left(x \leq\left[Y^{\prime}\right]\right.$ for some $Y^{\prime} \subset \subset Y$ with $[Y]=y)$. This will allow us to complete the proof of the assertion (i), and it will then be possible to deduce that the two relations $\subset \subset$ and $<<-$ the concrete and the abstract - are equivalent.

Let, then, a countably generated (right) Hilbert $\mathrm{C}^{*}$-module $X$ over $A$ be given. Choose, as follows, an increasing sequence

$$
X_{1} \subset \subset X_{2} \subset \subset \cdots \subset \subset X
$$

of subobjects, rapidly increasing in the concrete sense - that each subobject $X_{n}$ in the sequence is compactly contained in the next in what might be called the 
strong concrete sense - note that as pointed out above it follows that each $X_{n}$ is compactly contained in $X$ - such that $X$ is the subobject generated by $X_{1} \cup X_{2} \cup \cdots$, in other words such that the union is dense. In fact, that $X$ is countably generated is equivalent to the condition on the $\mathrm{C}^{*}$-algebra of compact endomorphisms of $X$ that it have a countable approximate unit (see Corollary 1.1.25 of [12]). (If $\left(\xi_{i}\right)$ is a generating sequence for $X$, with $\left\|\xi_{i}\right\|=2^{-i}$, then the endomorphism $\sum \xi_{i} \xi_{i}^{*}$ where $\xi_{i}^{*}$ denotes the module map $\xi \mapsto\left\langle\xi_{i},, \xi\right\rangle$ from $X$ to $A-$ and $\xi_{i} \xi_{i}^{*}$ the map $\xi \mapsto \xi_{i}\left\langle\xi_{i}, \xi\right\rangle$ !-is a strictly positive element of the $\mathrm{C}^{*}$-algebra of compact endomorphisms of $X$ : If $f$ is a positive functional zero on $\xi_{i} \xi_{i}^{*}$ for all $i$ then, since $\xi_{i} a a^{*} \xi_{i}^{*} \leq\|a\|^{2} \xi_{i} \xi_{i}^{*}$, the corresponding complex-valued inner product on $X$ is zero on $\xi_{i} A$ for every $i$ and therefore zero on all of $X$ - so $f$ is zero on every $\xi \xi^{*}$ and therefore zero. The converse is not needed and so we leave it to the reader; it can be proved by viewing the module as a subobject of the direct sum of a suitable number of copies of $A$.) Choose a countable approximate unit $\left(u_{n}\right)$ for the $\mathrm{C}^{*}$ algebra of compact endomorphisms of $X$ such that $u_{n+1} u_{n}=u_{n}$ for every $n$. Then the increasing sequence of subobjects

$$
X_{1}=\left(u_{1} X\right)^{-} \subseteq X_{2}=\left(u_{2} X\right)^{-} \subseteq \cdots \subseteq X
$$

has the required properties $\left(X_{n} \subset \subset X_{x+1}\right.$ and $\bigcup X_{n}$ dense in $\left.X\right)$.

By the case of the assertion (i) established above, $[X]=\sup \left[X_{n}\right]$ in the Cuntz (ordered) semigroup. In fact, in the proof of this an (apparently) stronger statement was obtained: if $Z \subset \subset X$ then $Z$ is isomorphic to a subobject of $X_{n}$ for some $n$, and hence for all sufficiently large $n$. It follows immediately that any two compactly contained subobjects of $X$ are isomorphic to subobjects of another compactly contained subobject of $X$, and (recall that $X_{n} \subset \subset X_{n+1}$ so that $Y \subseteq X_{n}$ implies $\left.Y \subset \subset X_{n+1}\right)$ in fact to compactly contained subobjects of such a subobject, and hence that in the Cuntz semigroup the set of elements $\subset \subset[X]$ is upward directed with respect to the relation $\subset \subset$. At the same time one sees that the supremum of this upward directed set of elements is $[X]$. (Note that this particular upward directed set of elements of the Cuntz semigroup contains a cofinal increasing sequence; in general, this is not the case, as can be seen by considering an uncountable direct sum of $\mathrm{C}^{*}$-algebras. One sees at the same time that an arbitrary upward directed subset of the Cuntz semigroup may not have a supremum.)

Now let us return to the general case of the first assertion of the theorem, i.e., that the sequence

$$
x_{1} \leq x_{2} \leq \cdots
$$


is an arbitrary increasing sequence in the Cuntz semigroup of $A$. Choose objects (countably generated Hilbert $\mathrm{C}^{*}$-modules) $X_{1}, X_{2}, \cdots$ representing the Cuntz equivalence classes $x_{1}, x_{2}, \cdots$ and as above for each $n=1,2, \cdots$ choose an increasing sequence of subobjects of $X_{n}$, each compactly contained in the next,

$$
X_{n 1} \subset \subset X_{n 2} \subset \subset \cdots \subset \subset X_{n}
$$

such that the sequence $X_{n 1}, X_{n 2}, \cdots$ generates $X_{n}$, so that as shown above

$$
\left[X_{n}\right]=\sup _{m}\left[X_{n m}\right]
$$

Now note that, as another instance of what was proved above, concerning the supremum of an increasing sequence of objects each contained compactly as a subobject of the next, for each $n$ and each $m$, there exists $r$ such that $X_{n m}$ is isomorphic to a compact subobject of $X_{n+1, r}$. We may therefore choose $m_{n}$ for each $n$ in such a way that

$$
\begin{aligned}
& {\left[X_{11}\right] \subset \subset\left[X_{2, m_{1}}\right]} \\
& {\left[X_{12}\right],\left[X_{2, m_{1}}\right] \subset \subset\left[X_{3, m_{2}}\right]} \\
& {\left[X_{13}\right],\left[X_{2, m_{1}+1}\right],\left[X_{3, m_{2}}\right] \subset \subset\left[X_{4, m_{3}}\right],}
\end{aligned}
$$

and, in general, for each $n$, the equivalence class $\left[X_{n+1, m_{n}}\right]$ compactly contains (in the concrete sense) $\left[X_{n, m_{n-1}}\right]$ and also one new term in each of the preceding sequences. In this way one obtains a rapidly increasing sequence of equivalence classes,

$$
\left[X_{1,1}\right] \subset \subset\left[X_{2, m_{1}}\right] \subset \subset\left[X_{3, m_{2}}\right] \subset \subset \cdots
$$

which eventually is greater than or equal to each fixed equivalence class $\left[X_{m n}\right]$, and each term of which is less than or equal to some term of the sequence $x_{1} \leq x_{2} \leq \cdots$. The supremum of this sequence, which exists by the special case of the assertion (i) established earlier, is therefore also the supremum of the sequence $x_{1} \leq x_{2} \leq \cdots$. (For each fixed $n$ it is greater than or equal to $x_{n}$ because it is greater than or equal to $\left[X_{n m}\right]$ for every $m$, and $x_{n}=\sup _{m}\left[X_{n m}\right]$.) This completes the proof of the assertion (i).

Let us now prove (using the assertion (i)) that the two notions of compact inclusion in the Cuntz semigroup, one defined concretely as the quotient relation arising from the inclusion up to isomorphism of all compactly contained subobjects of one countably generated Hilbert $\mathrm{C}^{*}$-module as subobjects of a fixed compactly contained subobject of another, written $\subset \subset$, and the other, written $<<$, defined purely order-theoretically, in other words, as may be done in any ordered set, by 
saying that one element is compactly contained in another if, whenever the larger one is less than or equal to the supremum of some increasing sequence (assumed to exist in the given case), the smaller one is already less than or equal to one of the terms in the sequence.

Let us show first that if $x \subset \subset y$ holds (the concrete relation) then $x<<y$ holds (the abstract relation). That $x \subset \subset y$ holds means that, with $y=[Y]$ (any choice of $Y), x \leq\left[Y^{\prime}\right]$ for some $Y^{\prime}$ with $Y^{\prime} \subset \subset Y$. Let $\left[Y_{1}\right] \leq\left[Y_{2}\right] \leq \cdots$ be such that $[Y] \leq \sup \left[Y_{n}\right]$, and let us show, as required, that $x \leq\left[Y_{n}\right]$ for some $n$.

Again, we shall use not so much the assertion (i) itself as its proof. Recall that in the proof of the existence of the supremum of an increasing sequence

$$
x_{1} \leq x_{2} \leq \cdots
$$

of equivalence classes in the Cuntz semigroup, a new increasing sequence

$$
x_{1}^{\prime} \leq x_{2}^{\prime} \leq \cdots
$$

of smaller elements (i.e., with $x_{1}^{\prime} \leq x_{1}, x_{2}^{\prime} \leq x_{2}, \cdots$ ) was constructed with the same supremum and such that the supremum could now be constructed as the inductive limit of an increasing sequence of objects,

$$
X_{1}^{\prime} \subseteq X_{2}^{\prime} \subseteq \cdots
$$

with $X_{n}^{\prime}$ representing $x_{n}^{\prime}$ for each $n$. Recall, furthermore, that it was proven in this special setting - when the supremum can be described as an inductive limitthat any compactly contained subobject of the inductive limit is isomorphic to a subobject of the finite-stage object $X_{n}^{\prime}$ for some $n$. (Note that this second statement was in fact used in the proof of the first statement; it is clearly of fundamental importance. It should perhaps be pointed out again that, from a technical point of view, this statement is essentially just Lemma 2.2 of [17].)

Accordingly, passing to a new sequence with smaller terms but the same supremum, instead of just considering a given sequence

$$
\left[Y_{1}\right] \leq\left[Y_{2}\right] \leq \cdots \leq \sup \left[Y_{n}\right]
$$

we may suppose that

$$
Y_{1} \subseteq Y_{2} \subseteq \cdots
$$

and $\sup \left[Y_{n}\right]=\left[\lim _{\rightarrow} Y_{n}\right]$. Then, as shown above (and just now recalled), if as assumed above, $x \subset \subset[Y]$ and $[Y] \leq \sup \left[Y_{n}\right]=\left[\lim _{\rightarrow} Y_{n}\right]$, so that in particular by definition of 
the relation $\subset \subset$ in the Cuntz semigroup, $x \leq\left[Y^{\prime}\right]$ for some $Y^{\prime} \subset \subset Y$, with therefore $Y^{\prime}$ isomorphic to some $Y^{\prime \prime} \subset \subset \lim Y_{n}$, then $Y^{\prime \prime}$ is isomorphic to a subobject of $Y_{n}$ for some $n$, and since $x \leq\left[Y^{\prime \prime}\right]$, therefore $x \leq\left[Y_{n}\right]$ as desired.

Conversely, let us show that if $x<<y$ holds (the abstract relation, defined just in terms of the order relation $\leq$ ) then $x \subset \subset y$ holds (the concrete relation), i.e., $x \leq\left[Y^{\prime}\right]$ for some $Y^{\prime} \subset \subset Y$ with $[Y]=y$. By the assertion (ii), with the relation $<<$ replaced by the relation $\subset \subset$-in which form this assertion has now been proved-, as shown above we may express a given element $y$ of the Cuntz semigroup as the equivalence class of the inductive limit of a rapidly increasing sequence of objects, say

$$
Y_{1} \subset \subset Y_{2} \subset \subset \cdots
$$

Then, with $Y=\lim _{\rightarrow} Y_{n}$, so that $[Y]=y$, if $x<<y$ then, by definition, $x \leq\left[Y_{n}\right]$ for some $n$. By construction, $Y_{n} \subset \subset Y$, as desired.

Before proceeding to the proof of the last assertion of the theorem, concerning the very strong compatibility of the order relation on Cuntz equivalence classes with addition, we must return to the problem of the very basic compatibility of the pre-order relation on (isomorphism classes of ) countably generated Hilbert $\mathrm{C}^{*}$ modules with addition, put aside at the beginning of the proof. The problem was reduced to the question of showing that if $X \subset \subset X_{1} \oplus X_{2}$ then $X$ is isomorphic to a subobject of $X_{1}^{\prime} \oplus X_{2}^{\prime}$ for some compactly contained subobjects $X_{1}^{\prime} \subset \subset X_{1}$ and $X_{2}^{\prime} \subset \subset X_{2}$. Express $X_{1}$ and $X_{2}$, as described above, as the closure of the union of rapidly increasing sequences of subobjects

$$
X_{1}^{1} \subset \subset X_{1}^{2} \subset \subset \cdots \subset X_{1} \text { and } X_{2}^{1} \subset \subset X_{2}^{2} \cdots \subset \subset X_{2}
$$

and note that then $X_{1} \oplus X_{2}$ is the closure of the union of the rapidly increasing sequence of subobjects

$$
X_{1}^{1} \oplus X_{2}^{1} \subset \subset X_{1}^{2} \oplus X_{2}^{2} \subset \subset \cdots \subset \subset X_{1} \oplus X_{2}
$$

As shown above, $\left[X_{1} \oplus X_{2}\right]$ is then the supremum of the increasing sequence $\left(\left[X_{1}^{n} \oplus X_{2}^{n}\right]\right)$ in the ordered set of Cuntz equivalence classes. As also shown above, the relation $\subset \subset$ between modules implies the purely order-theoretic relation $<<$ between their Cuntz equivalence classes, and so, since $X \subset \subset X_{1} \oplus X_{2}$, we have $[X]<<\left[X_{1} \oplus X_{2}\right]$. Hence,

$$
[X] \leq\left[X_{1}^{n} \oplus X_{2}^{n}\right]
$$


for some $n=1,2, \cdots$. Since (again cf. above) there exists a subobject $X^{\prime}$ of $X_{1} \oplus X_{2}$ with

$$
X \subset \subset X^{\prime} \subset \subset X_{1} \oplus X_{2}
$$

we may conclude by replacing $X$ by $X^{\prime}$ above that

$$
\left[X^{\prime}\right] \leq\left[X_{1}^{n} \oplus X_{2}^{n}\right]
$$

(for some $n$ ), from which it follows, by definition, that $X$ is isomorphic to a subobject of $X_{1}^{n} \oplus X_{2}^{n}$ (compactly contained, but we don't need this), and so $X_{1}^{n}$ and $X_{2}^{n}$ fulfil the requirements for $X_{1}^{\prime}$ and $X_{2}^{\prime}$.

At this point we could also adduce another proof of additivity of the order relation $\leq$ on Cuntz equivalence classes, by showing that the relation $[X] \leq[Y]$ as defined in the present article is equivalent to the approximative comparison relation considered by Cuntz (from which additivity would be immediate, just as for the stronger order relation that one module is isomorphic to a submodule of another). (Incidentally, by Theorem 3 below this last, extremely simple, (pre)-order relation is the same as the Cuntz pre-order relation on countably generated Hilbert $\mathrm{C}^{*}$ modules in the case that the $\mathrm{C}^{*}$-algebra has stable rank one-and, as mentioned above, it is antisymmetric - i.e., an order relation-on isomorphism classes.) This approximative order relation is most easily stated in the present context in terms of compact homomorphisms: There should exist a compact homomorphism from $Y$ to $X$ with image containing approximants to a given finite subset of $X$. An alternative formulation is that there should exist a compact homomorphism from $X$ to $Y$ approximately preserving the norms of a given finite set of elements of $X$. It is in fact immediate from the fact that any finite subset of $X$ is approximately contained in a compactly contained subobject (shown above) that both types of maps exist (from $Y$ to $X$ and from $X$ to $Y$ ), when $[X] \leq[Y]$, - and that these may be chosen to be contractions. (Just use that for any compactly contained subobject, of either $X$ or $Y$ (of $Y$ to obtain a map to $X$, and of $X$ to obtain a map to $Y$ ), there exists a compact contraction into this subobject which is approximately the identity on it.) That the existence of one or the other kind of map (from $Y$ to $X$ or from $X$ to $Y$ ) implies $[X] \leq[Y]$ follows from Lemma 2.2 of [17]. (The present notion of comparability is shown to be equivalent to Cuntz's one in Appendix 6, below.)

To deal with the final part of the statement of the theorem, let us show first that $\sup \left(S_{1}+S_{2}\right)=\sup S_{1}+\sup S_{2}$ if $S_{1}$ and $S_{2}$ are countable upward directed subsets of the Cuntz semigroup of $A$. As we have shown, $\sup S_{1}$ and $\sup S_{2}$ may 
both be represented by the inductive limits of increasing sequences of countably generated Hilbert $\mathrm{C}^{*}$-modules - the equivalence classes of which are each less than or equal to the equivalence class of some element of $S_{1}$ or $S_{2}$, respectively. Clearly, the direct sum of these inductive limits is the inductive limit of the direct sums, and we have also shown that the inductive limit of any increasing sequence of countably generated Hilbert $\mathrm{C}^{*}$-modules gives rise to the supremum in the Cuntz semigroup. (Both these statements were established in the course of the proof of the assertion (i).) The statement follows (given that, in the Cuntz semigroup, the relation $\leq$ is compatible with addition - in other words, one has an ordered semigroup).

Finally, we must show that the relation $<<$ of compact containment in the order-theoretic sense is compatible with addition, in other words, that if $x_{1}<<y_{1}$ and $x_{2}<<y_{2}$, then $x_{1}+x_{2}<<y_{1}+y_{2}$. This is seen immediately to hold with the relation $\subset \subset$ in place of the relation $<<$ (given that the Cuntz semigroup is an ordered semigroup), and we have proved above that these two relations are equivalent.

2. Let us denote by $\mathcal{C} u$ the category of ordered abelian semigroups with the properties established in Theorem 1, with, as maps, semigroup maps preserving the zero element, preserving suprema of countable upward directed subsets, and preserving the relation $<<$ of compact containment in the order-theoretic sense.

Theorem. The Cuntz semigroup is a functor from the category of $C^{*}$-algebras, with *-homomorphisms as maps, to the category $\mathcal{C} u$, preserving inductive limits of sequences - which always exist in the category $\mathcal{C} u$ (as well as in the category of $C^{*}$-algebras).

Proof. Let us first show that sequential inductive limits exist in the category $\mathcal{C} u$. Let $S_{1} \rightarrow S_{2} \rightarrow \ldots$ be a sequence in the category $\mathcal{C} u$. In order to construct the inductive limit of this sequence let us first show that the collection of increasing sequences $\left(s_{1}, s_{2}, \cdots\right)$ with $s_{1} \in S_{1}, s_{2} \in S_{2}, \cdots$-increasing in the sense that for each $i$ the image of $s_{i} \in S_{i}$ in $S_{i+1}$ is less than or equal to $s_{i+1}$-becomes a preordered abelian semigroup with the addition operation

$$
\left(s_{i}\right)+\left(t_{i}\right)=\left(s_{i}+t_{i}\right)
$$

and the pre-order relation

$\left(s_{i}\right) \leq\left(t_{i}\right)$ if for any $i$ and any $s \in S_{i}$ with $s<<s_{i}$, eventually $s<<t_{j}$ (in $S_{j}$ ).

Note that addition makes sense: if the sequences $\left(s_{i}\right)$ and $\left(t_{i}\right)$ with $s_{i}, t_{i} \in S_{i}$ are increasing, i.e., if $s_{i} \leq s_{i+1}$ and $t_{i} \leq t_{i+1}$ in $S_{i+1}$ for every $i$, then by compatibility of the order relation with addition in $S_{i+1}$, also $s_{i}+t_{i} \leq s_{i+1}+t_{i+1}$ for every $i$, so 
that the sequence $\left(s_{i}+t_{i}\right)$ belongs to the collection considered. That this collection becomes an abelian semigroup with this addition follows immediately from the fact that each $S_{i}$ and hence also the Cartesian product $\Pi S_{i}$ is an abelian semigroup (in other words that addition in $S_{i}$ is associative and commutative). We must check that the relation $\left(s_{i}\right)<<\left(t_{i}\right)$ is a pre-order relation, i.e., is reflexive and transitive. It is reflexive because if $\left(s_{i}\right)$ is an increasing sequence with $s_{i} \in S_{i}$ and if $s<<s_{i}$ for some $i$, then $s<<s_{j}$ for all $j \geq i$ (as both $s<<s_{i}$ and $s_{i} \leq s_{j}$ in $S_{j}$ for all $j \geq i$, because the maps in the sequence $S_{1} \rightarrow S_{2} \rightarrow \cdots$ preserve both the relations $\leq$ and $<<$, and since by definition if $x<<y$ and $y \leq z$ in an ordered set then $x<<z$ ). It is transitive as an immediate consequence of the definition (if increasing sequences $\left(s_{i}\right),\left(t_{i}\right)$, and $\left(u_{i}\right)$, with $s_{i}, t_{i}, u_{i} \in S_{i}$, are given, such that $\left(s_{i}\right) \leq\left(t_{i}\right)$ and $\left(t_{i}\right) \leq\left(u_{i}\right)$, then for any $i$ and any $s \in S_{i}$ with $s<<s_{i}$, first, eventually $s<<t_{j}$, and in particular $s<<t_{j}$ for some $j$ (this is in fact the same thing), and, hence, second, eventually $s<<u_{k}$, as is needed to show $\left.\left(s_{i}\right) \leq\left(u_{i}\right)\right)$. Finally, we must check that this pre-order relation is compatible with addition, i.e., that if $\left(s_{i}\right),\left(s_{i}^{\prime}\right)$ and $\left(t_{i}\right),\left(t_{i}^{\prime}\right)$ are increasing sequences with $s_{i}, s_{i}^{\prime}, t_{i}, t_{i}^{\prime} \in S_{i}$ and with $\left(s_{i}\right) \leq\left(s_{i}^{\prime}\right)$ and $\left(t_{i}\right) \leq\left(t_{i}^{\prime}\right)$, then $\left(s_{i}+t_{i}\right) \leq\left(s_{i}^{\prime}+t_{i}^{\prime}\right)$. Let $s \in S_{i}$ for some $i$ be such that $s<<s_{i}+t_{i}$ and let us show that eventually $s<<s_{j}^{\prime}+t_{j}^{\prime}$. Choose increasing sequences

$$
s_{i}^{1} \leq s_{i}^{2} \leq \cdots<<s_{i} \text { and } t_{i}^{1} \leq t_{i}^{2} \leq \cdots<<t_{i}
$$

in $S_{i}$ with suprema $s_{i}$ and $t_{i}$ respectively - these exist by hypothesis (even rapidly increasing, but we do not need the full force of this). Then as by hypothesis the relation $\leq$ and the operation of passing to the supremum of an increasing sequence are compatible with addition in $S_{i}$, we have

$$
s_{i}^{1}+t_{i}^{1} \leq s_{i}^{2}+t_{i}^{2} \leq \cdots \leq s_{i}+t_{i}
$$

and furthermore $s_{i}+t_{i}=\sup _{n}\left(s_{i}^{n}+t_{i}^{n}\right)$. Hence as $s<<s_{i}+t_{i}$, for $n$ sufficiently large, $s \leq s_{i}^{n}+t_{i}^{n}$. Since $s_{i}^{n}<<s_{i}$ and $t_{i}^{n}<<t_{j}^{1}$, it follows from $\left(s_{k}\right) \leq\left(s_{k}^{\prime}\right)$ and $\left(t_{k}\right) \leq\left(t_{k}^{\prime}\right)$ that for sufficiently large $j$ (with $i$ fixed as above) also $s_{i}^{n}<<s_{j}^{\prime}$ and $t_{i}^{n}<<t_{j}^{\prime}$. Hence by compatibility of the relation $<<$ with addition, for large $j$

$$
s_{i}^{n}+t_{i}^{n} \leq s_{j}^{\prime}+t_{j}^{\prime}
$$

and so, combining this with $s \leq s_{i}^{n}+t_{i}^{n}$, we have $s \leq s_{j}^{\prime}+t_{j}^{\prime}$ as desired. (It is immediate from the definition of the relation $<<$ in an ordered set that if $x \leq y$ and $y<<z$ then $x<<z$-for that matter also that if $x<<y$ and $y \leq z$ then $x \leq z$, and we shall also use this, in the very next step.) 
Let us now show that the quotient of the pre-ordered abelian semigroup just defined by the equivalence relation derived from the pre-order-i.e., $s$ equivalent to $t$ if $s \leq t$ and $t \leq s$-is an ordered abelian semigroup belonging to $\mathcal{C} u$, and furthermore is the inductive limit in this category of the sequence $S_{1} \rightarrow S_{2} \rightarrow \cdots$.

Denote this ordered abelian semigroup by $S$. To show that $S$ belongs to the category $\mathcal{C} u$ we must show that $S$ has a zero element, that each increasing sequence in $S$ has a supremum (equivalently, each countable upward direct set has a supremum) that each element of $S$ is the supremum of a rapidly increasing sequence (each term compactly contained in the next in the order-theoretic sense), and, finally, that the relations $\leq$ and $<<$ and the operation of passing to the supremum of an increasing sequence are compatible with addition.

The sequence $(0,0, \cdots)$ - or, rather, its equivalence class-is a zero element. (Necessarily unique.) In order to establish the other desired properties of $S$ it is convenient to show first that every increasing sequence $\left(s_{1}, s_{2}, \cdots\right)$ with $s_{i} \in S_{i}$ is equivalent to a rapidly increasing one. This is done by choosing for each $i$ a rapidly increasing sequence in $S_{i}$ with supremum $s_{i}$ (which exists by hypothesis), and then passing to a subsequence of each of these sequences, one after another, starting with the second one, using the compact containment of each term in the sequence for $s_{i}$ in $s_{i}$, to ensure that, for each $i$, each term of the sequence for $s_{i+1}$ is greater than or equal to the corresponding term of the sequence for $s_{i}$. Then the Cantor diagonal sequence - the $i$ th term of which is the $i$ th term of the (new) sequence for $s_{i}$-is rapidly increasing and equivalent to the sequence $\left(s_{i}\right)$. The $(i+1)$ st term of this sequence, which is the $(i+1)$ st term of the sequence for $s_{i+1}$, majorizes the $(i+1)$ st term of the sequence for each of $s_{1}, \cdots, s_{i}$, and, in particular, as the $(i+1)$ st term of the sequence for $s_{i}$ compactly contains the $i$ th term of this sequence, which is the $i$ th term of the diagonal sequence, it follows that the $i$ th term of the diagonal sequence is compactly contained in the $(i+1)$ st term. Since the $i$ th term of the diagonal sequence is less than or equal to $s_{i}$, and (for the second, but not the last, time) since $x<<y$ and $y \leq z$ implies $x<<z$, if an element $s$ of $S_{i}$ is compactly contained in the $i$ th term of the diagonal sequence (an element of $S_{i}$ ), then it is compactly contained in $s_{i}$ (in $S_{i}$, and hence by preservation of compact containment also in $S_{j}$ for $j \geq i$, and hence it is also compactly contained in $s_{j}$ for $j \geq i$ ). To prove that the diagonal sequence is equivalent to $\left(s_{i}\right)$ it remains to show that if $s<<s_{i}$ for some $s \in S_{i}$ then $s$ is compactly contained in all except finitely many terms of the diagonal sequence. Choose $j$ such that the $j$ th term of the sequence chosen for $s_{i+1}$ is greater than or equal to $s$; such $j$ exists by the definition of compact containment, as the supremum of this sequence, i.e., $s_{i+1}$, is 
greater than or equal to $s_{i}$, also in $S_{i+1}$, and so compactly contains $s$.

Let us now show that each increasing sequence in $S$ has a supremum. If $s^{1} \leq$ $s^{2} \leq \cdots$ is an increasing sequence in $S$, by what we have just shown there exist rapidly increasing sequences $\left(s_{n}^{1}\right),\left(s_{n}^{2}\right), \cdots$, with $s_{n}^{i} \in S_{n}$, representing $s^{1}, s^{2}, \cdots$. Passing to subsequences very much as above we may suppose that

$$
s_{i}^{1}<<s_{i}^{2}<<\cdots
$$

for each $i$, from which it follows, first (immediately), that the diagonal sequence is rapidly increasing, i.e.,

$$
s_{1}^{1}<<s_{2}^{2}<<\cdots,
$$

with the $n$th inequality holding in $S_{n+1}$, and, second, as we shall now show, that this sequence represents the supremum of $s^{1}, s^{2}, \cdots$ in $S$. To see that the class $s$ of $\left(s_{1}^{1}, s_{2}^{2}, \cdots\right)$ is the supremum of $s^{1}, s^{2}, \cdots$ in $S$, note first that, for each $i$ and each $n, s_{n}^{i} \leq s_{k}^{k}$ where $k=\max (i, n)$, whence $s^{i} \leq s$, and, second, that if $t=\left(t_{1}, t_{2}, \cdots\right) \geq s^{1}, s^{2}, \cdots$, then for each $i$, if $r<<s_{i}^{i}$ in $S_{i}$ then, as $s_{i}^{i}$ is the $i$ th term of $s^{i}$, eventually $r<<t_{j}$-and so $s \leq t$.

Let us show next that each element of $S$ is the supremum of a rapidly increasing sequence. By what we have shown above, a given element $s$ of $S$ is represented by a rapidly increasing sequence $\left(s_{i}\right)$ with $s_{i} \in S_{i}$, i.e., a sequence with $s_{1}<<s_{2}<<\cdots$ (not just $s_{1} \leq s_{2} \leq \cdots$ ). The sequence

$$
\left(s_{1}, s_{1}, \cdots\right),\left(s_{1}, s_{2}, s_{2}, \cdots\right),\left(s_{1}, s_{2}, s_{3}, s_{3}, \cdots\right), \cdots
$$

in $S$ is then rapidly increasing and has supremum $s$. (It is rapidly increasing because $s_{i}<<s_{i+1}$, not only in $S_{i+1}$ but also in $S_{j}$ for $j \geq i+1$-note that preservation of compact containment by morphisms has already been used above. To see that the supremum of the sequence is equal to $s$, let $t \in S$ be given with

$$
t \geq\left(s_{1}, s_{1}, \cdots\right),\left(s_{1}, s_{2}, s_{2}, \cdots\right), \cdots
$$

and let us prove that $t \geq s$. Choose just any (increasing) sequence $\left(t_{i}\right)$, with $t_{i} \in S_{i}$, representing $t$. For each $i$, we have $s_{i}<<s_{i+1}$ in $S_{i+1}$ and hence, as $\left(s_{1}, \cdots, s_{i}, s_{i+1}, s_{i+1}, \cdots\right) \leq t, s_{i}<<t_{j}$ in $S_{j}$ for all sufficiently large $j$. This shows that $\left(s_{1}, s_{2}, s_{3}, \cdots\right) \leq\left(t_{1}, t_{2}, t_{3}, \cdots\right)$, and so $s \leq t$ in the quotient ordered set $S$.

Next, let us show that the relations $\leq$ and $<<$ and the operation of passing to the supremum of an increasing sequence in $S$ are compatible with addition. First, recall what we have shown above, that any single element of $S$ can be represented 
by a rapidly increasing sequence $\left(s_{1}, s_{2}, \cdots\right)$ with $s_{i} \in S_{i}$, and that, furthermore, for any increasing sequence $\left(s^{i}\right)$ in $S$ with supremum $s$ there is a rapidly increasing sequence $\left(s_{1}, s_{2}, \cdots\right)$, with $s_{i} \in S_{i}$, representing $s$, such that $s_{i} \leq s^{i}$ for every $i$, so that, in particular, $\sup s_{i}=\sup s^{i}$; note that it follows from the definition of the order relation on $S$ that $\sup s_{i}=s$ for any rapidly increasing sequence $\left(s_{1}, s_{2}, \cdots\right)$ with $s_{i} \in S_{i}$ representing $s$ (indeed, the construction of such a representing sequence above shows that this is true even for a representing increasing sequence which is not rapidly increasing). In particular, choosing such representing sequences $\left(s_{1}, s_{2}, \cdots\right)$ and $\left(t_{1}, t_{2}, \cdots\right)$ for the suprema $s$ and $t$ of two increasing sequences $\left(s^{i}\right)$ and $\left(t^{i}\right)$ in $S$, note that $\left(s_{1}+t_{1}, s_{2}+t_{2}, \ldots\right)$ is a representing sequence for $s+t$ with analogous properties - rapidly increasing, and with $s_{i}+t_{i} \leq s^{i}+t^{i}$ - where we do not assume that $\sup \left(s^{i}+t^{i}\right)=s+t$, but we may now compute as follows:

$$
s+t=\sup \left(s_{i}+t_{i}\right) \leq \sup \left(s^{i}+t^{i}\right) \leq \sup s^{i}+\sup t^{i}=s+t,
$$

which proves that

$$
\sup \left(s^{i}+t^{i}\right)=\sup s^{i}+\sup t^{i}
$$

so that we have proved that taking suprema is compatible with addition.

¿From the compatibility of the operation of taking suprema of increasing sequences in $S$ with addition, the compatibility of the relation $\leq$ with addition follows. Indeed, if $s^{1} \leq s^{2}$ and $t^{1} \leq t^{2}$ in $S$ then, choosing rapidly increasing representative sequences $\left(s_{i}^{1}\right),\left(s_{i}^{2}\right),\left(t_{i}^{1}\right)$, and $\left(t_{i}^{2}\right)$ for $s^{1}, s^{2}, t^{1}$, and $t^{2}$, with $s_{i}^{1}, s_{i}^{2}, t_{i}^{1}$ and $t_{i}^{2} \in S_{i}$ (actually, it is enough for the representing sequences for $s^{1}$ and $t^{1}$ just to be increasing), we may replace $\left(t_{i}^{1}\right)$ and $\left(t_{i}^{2}\right)$ by subsequences in such a way that $s_{i}^{1} \leq s_{i}^{2}$ and $t_{i}^{1} \leq t_{i}^{2}$ for every $i$, and then we have

$$
\begin{aligned}
s^{1}+t^{1} & =\sup s_{i}^{1}+\sup t_{i}^{1} \\
& =\sup \left(s_{i}^{1}+t_{i}^{1}\right) \\
& \leq \sup \left(s_{i}^{2}+t_{i}^{2}\right) \\
& \leq \sup s_{i}^{2}+\sup t_{i}^{2} \text { (Equality not needed here.) } \\
& =s^{2}+t^{2}
\end{aligned}
$$

i.e., $s^{1}+t^{1} \leq s^{2}+t^{2}$, as desired.

The compatibility of $<<$ with addition is simpler. If $s^{1}<<s^{2}$ and $t^{1}<<t^{2}$ in $S$ then, choosing rapidly increasing representing sequences $\left(s_{i}^{2}\right)$ and $\left(t_{i}^{2}\right)$, with $s_{i}^{2}, t_{i}^{2} \in S_{i}$, from $s^{1}<<s^{2}$ and $t^{1}<<t^{2}$ we deduce that eventually $s^{1} \leq s_{i}^{2}$ and $t^{1} \leq t_{i}^{2}$ and so, eventually,

$$
s^{1}+t^{1} \leq s_{i}^{2}+t_{i}^{2}<<s_{i+1}^{2}+t_{i+1}^{2} \leq s^{2}+t^{2},
$$


i.e., $s^{1}+t^{1}<<s^{2}+t^{2}$, as desired.

Now let us complete the proof of the purely order-theoretic part of the theorem by proving that the object $S$ of the category $\mathcal{C} u$ constructed above is the inductive limit in this category of the given sequence $S_{1} \rightarrow S_{2} \rightarrow \cdots$. We must show that for every object $T$ in $\mathcal{C} u$ and every compatible sequence of maps $S_{1} \rightarrow T, S_{2} \rightarrow T, \cdots$ there exists a unique compatible map $S \rightarrow T$ :

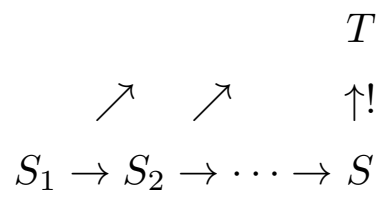

Of course, for this to make sense we must have maps $S_{i} \rightarrow S$ for all $i$ compatible with the maps $S_{i} \rightarrow S_{i+1}$. For each $i$, and each $s \in S_{i}$, note that the sequence $(0, \cdots, s, s, \cdots)$, with 0 until the $(i-1)$ st term and then $s$ from the $i$ th term on, is increasing and therefore represents an element of $S$. For each fixed $i$ and $s \in S_{i}$, note that for any $j \geq i$ the sequence with 0 up to the $(j-1)$ st term and $s$ from the $j$ th term on is equivalent to the one with $j=i$, defined above; this follows immediately from the definition of equivalence of increasing sequences with $k$ th term in $S_{k}$ for all $k$. This shows that the maps $S_{1} \rightarrow S, S_{2} \rightarrow S, \cdots$ obtained in this way are compatible with the given sequence $S_{1} \rightarrow S_{2} \rightarrow \cdots$ (i.e., that when adjoined to it they yield a commutative diagram).

The definition of a (set) map $S \rightarrow T$ is immediate if one restricts to rapidly increasing representative sequences for elements of $S$ (shown above always to exist). (If $\left(s_{1}, s_{2}, \ldots\right)$ represents $s$ with $s_{i} \in S_{i}$ and $s_{1}<<s_{2}<<\cdots$, let us aim to map $s$ into the supremum of the increasing sequence in $T$ consisting of the images of $s_{1}, s_{2}, \cdots$ by the maps $S_{1} \rightarrow T, S_{2} \rightarrow T \cdots$; this of course makes sense if the sequence $\left(s_{1}, s_{2}, \cdots\right)$ is just increasing. If $\left(s_{1}^{\prime}, s_{2}^{\prime}, \ldots\right)$ is a second rapidly increasing representative of $s$ then, as $\left(s_{i}\right)$ is rapidly increasing, for each $i$ we have $s_{i}<<$ $s_{i+1} \leq s=\sup s_{i}^{\prime}$ and so eventually $s_{i} \leq s_{j}^{\prime}$; by symmetry, also each $s_{i}^{\prime}$ is eventually $\leq s_{j}$, and so the suprema of the images of $s_{1}, s_{2}, \cdots$ and of $s_{1}^{\prime}, s_{2}^{\prime}, \cdots$ in $T$ are equal (as the maps $S_{i} \rightarrow T$ preserve the order relation and are compatible as set maps).)

Let us check that the map $S \rightarrow T$ thus defined is compatible with the maps $S_{i} \rightarrow T$ (i.e., that the diagram is commutative as a diagram of set maps), that it is a morphism in the category $\mathcal{C} u$, and that it is unique with these properties.

To show compatibility of $S_{i} \rightarrow T$ with $S \rightarrow T$, we must show, for each fixed $i$, that if $s \in S_{i}$ then the image of $s$ in $T$ by the given map $S_{i} \rightarrow T$ is the same as 
the image of $s$ in $T$ by the composed map $S_{i} \rightarrow S \rightarrow T$. By definition, the image of $s$ in $S$ is represented by the sequence $(0, \cdots, s, s, \cdots)$ consisting of the element $s$ repeated beginning with the $i$ th coordinate; however, in order to compute the image of this element of $S$ in $T$ we must represent it by a rapidly increasing sequence: let us use the sequence $\left(0, \cdots, r_{i}, r_{i+1}, \cdots\right)$-i.e., $r_{j}$ in the $j$ th place for $j \geq i$-where $\left(r_{j}\right)$ is a rapidly increasing sequence in $S_{i}$ with supremum $s$. By definition the corresponding element in $T$ is the supremum of the (increasing sequence of) images of the elements $r_{i} \in S_{i}, r_{i+1} \in S_{i+1}, \cdots$ by the maps $S_{i} \rightarrow T, S_{i+1} \rightarrow T, \cdots$, equivalently (by commutativity) of the images of $r_{i}, r_{i+1}, \cdots \in S_{i}$ by the map $S_{i} \rightarrow T$, and as this map preserves increasing sequential suprema, the supremum in $T$ in question is just the image of $s$, by the map $S_{i} \rightarrow T$, as desired.

To show that the map $S \rightarrow T$ belongs to the category $\mathcal{C} u$, we must show that it preserves addition, preserves the order relation, preserves suprema of increasing sequences, and preserves the order-theoretic relation $<<$ defined earlier, in terms of the two notions just mentioned (the order relation $\leq$ and the operation of sequential increasing supremum). Let us address these issues, briefly, in turn.

Given two rapidly increasing sequences $\left(r_{1}, r_{2}, \cdots\right)$ and $\left(s_{1}, s_{2}, \cdots\right)$ with $r_{i}, s_{i} \in$ $S_{i}$ for each $i$, to check that the sum in $S$ maps into the sum of the images in $T$ it is enough to recall what these images are, and that the operation of passing to the supremum of an increasing sequence in $T$ is compatible with addition in $T$. To check that the relation $\left(r_{1}, r_{2}, \cdots\right) \leq\left(s_{1}, s_{2}, \cdots\right)$ in $S$ (with $\left(r_{i}\right),\left(s_{i}\right)$ rapidly increasing as above) leads to the same relation between the images in $T$, recall that the equivalence relation defining $S$ is just derived from the pre-order relation between sequences which leads to the order relation in $S$ - and we have already shown that the map $S \rightarrow T$ exists! (And this by the only way conceivable, namely, by just proving that the pre-order is preserved.)

To check that the map from $S$ to $T$ preserves suprema of increasing sequences, recall from the proof that such suprema exist in $S$, given above, that representatives of an increasing sequence of elements of $S$ may be chosen in such a way that, not only is each representing sequence rapidly increasing, but also, the sequence of $i$ th terms for each fixed $i$ is rapidly increasing - and then the diagonal sequence is also rapidly increasing and furthermore represents the supremum of the given increasing sequence in $S$. Recalling the definition of the map $S \rightarrow T$ one sees immediately then that the image of the supremum is the supremum of the images. (The images of the terms of the diagonal sequence eventually majorize the images of the terms of each of the representing sequences, and so their supremum majorizes the supremum of the suprema - of the images of the terms of the individual representing sequences.) 
Finally, to check that the map $S \rightarrow T$ is compatible with the relation $<<$, let $\left(r_{1}, r_{2}, \cdots\right)$ and $\left(s_{1}, s_{2}, \cdots\right)$ be rapidly increasing representing sequences as above (i.e., with $r_{i}, s_{i} \in S_{i}$ ), and suppose that $\left(r_{i}\right)<<\left(s_{i}\right)$; we must show that the supremum of the images of $r_{1}, r_{2}, \cdots$ in $T$ is compactly contained (of course in the order-theoretic sense) in the supremum of the images of $s_{1} s_{2}, \cdots$-in other words, that, if $\dot{r}$ denotes the image of $r=\sup r_{i}$ in $T$, and $\dot{s}$ the image of $s=\sup s_{i}$ in $T$, then $\dot{r}<<\dot{s}$. The proof is very simple: since $s=\sup s_{i}$ and $r<<s$, we have $r \leq s_{i}$ for some $i$. Since the map $S \rightarrow T$ is already known to preserve the relation $\leq$, it follows that $\dot{r} \leq \dot{s}$ in $T$. Since $s_{i}<<s_{i+1}$, not only in $S$ but also in $S_{i+1}$, it follows from the properties of the given map $S_{i+1} \rightarrow T$ that $\dot{s}_{i}<<\dot{s}_{i+1}$ in $T$. Again, since $s_{i+1} \leq s$ in $S$, we have $\dot{s}_{i+1} \leq \dot{s}$ in $T$, and hence $\dot{s}_{i}<<\dot{s}$ in $T$, and hence also $\dot{r}<<\dot{s}$ in $T$, as desired.

Finally, let us show that the association of the Cuntz ordered semigroup to a $\mathrm{C}^{*}$ algebra gives rise in a natural way to a functor from the category of all $\mathrm{C}^{*}$-algebras to $\mathcal{C} u$, and that this functor preserves inductive limits of sequences.

By the functor's arising naturally we just mean that we have still to describe the morphism between the Cuntz semigroups that should correspond to a morphism between $\mathrm{C}^{*}$-algebras. Given $\mathrm{C}^{*}$-algebras $A$ and $B$ and $\mathrm{C}^{*}$-algebra morphism $A \rightarrow B$ (a map preserving the ${ }^{*}$-algebra structure - necessarily a contraction), to each given Hilbert $\mathrm{C}^{*}$-module $X=X_{A}$ over $A$, associate the Hilbert $\mathrm{C}^{*}$-module over $B$ defined by completing the (right) $B$-module $\left(X_{A}\right) \otimes_{A}\left({ }_{A} B\right)$ with respect to the (possibly degenerate) $B$-valued inner product

$$
\left\langle\sum \xi_{i} \otimes b_{i}, \sum \xi_{j}^{\prime} \otimes b_{j}^{\prime}\right\rangle_{B}=\sum_{i, j} b_{i}^{*}\left\langle\xi_{i}, \xi_{j}^{\prime}\right\rangle_{A} b_{j}^{\prime}
$$

where $B$ is considered as a left $A$-module by virtue of the given homomorphism $A \rightarrow B$.

Note that this correspondence, from Hilbert $A$-modules to Hilbert $B$-modules, takes countably generated Hilbert $A$-modules to countably generated Hilbert $B$ modules. Let us show that it preserves (in a natural way) the relation of inclusion as subobject, and also preserves the relation of compact inclusion-i.e., inclusion as a compactly contained subobject. Since it clearly preserves the relation of isomorphism (in a natural way), it follows that it preserves the Cuntz pre-order relation (as defined above - i.e., compactly contained subobjects of the first of two objects isomorphic to compactly contained subobjects of the second).

In fact it is also clear that a morphism $X \rightarrow Y$ of Hilbert $A$-modules, by which let us mean one preserving the $A$-valued inner product, is transformed by the push- 
forward construction described above into a morphism $X_{B} \rightarrow Y_{B}$ (of Hilbert $B$ modules). (At the purely algebraic level of the construction it is immediate that the natural push-forward maps $\left(X_{A}\right) \otimes\left({ }_{A} B\right) \rightarrow\left(Y_{A}\right) \otimes\left({ }_{A} B\right)$ (tensor products over $A$ ) preserves the $B$-valued inner product, and hence is isometric (although at this stage the norms may be seminorms), and then it follows by continuity that this holds for the extension to the completion. (One does not need this but note that by Theorem 3.5 of [15]) it is enough to note that the extension is isometric, and a $B$-module map, as this by itself implies preservation of the $B$-valued inner product.)

Let, then, $X_{A}$ be compactly contained in $Y_{A}$, and let us show that the pushed forward inclusion of $X_{B}$ in $Y_{B}$ is a compact one, i.e., that there is a compact selfadjoint endomorphism of $Y_{B}$ that acts as the identity on $X_{B} \subseteq Y_{B}$. The hypothesis is that there exists a compact self-adjoint endomorphism, say $t$, of $Y_{A}$ which acts as the identity on $X_{A} \subseteq Y_{A}$. It is sufficient to show that the endomorphism $t=t_{A}$ has a push-forward, in the natural sense, to a compact self-adjoint endomorphism $t_{B}$ of $Y_{B}$ (putting $X$ aside completely). The natural property that $t_{B}$ should have is of course that $t_{B}(\eta \otimes b)=\left(t_{A} \eta\right) \otimes b$, for $\eta \in Y_{A}$ and $b \in B$. This condition determines purely algebraically a map on $\left(Y_{A}\right) \otimes_{A}\left({ }_{A} B\right)$, which is bounded because $\left\langle t_{A} \eta, t_{A} \eta\right\rangle_{A} \leq\left\|t_{A}^{*} t_{A}\right\|\langle\eta, \eta\rangle_{A}$ (and the fact that this also holds when $\eta$ is replaced by $\left.\eta_{1} \otimes \cdots \otimes \eta_{k} \in Y_{A} \otimes \cdots \otimes Y_{A}\right)$. This shows that any adjointable endomorphism of $Y_{A}$ can be pushed forward to $Y_{B}$; that the push-forward, $t_{B}$, of $t_{A}$ is compact if $t_{A}$ is follows from the fact that this is clear (purely algebraically) if $t_{A}$ is of finite rank (i.e., a finite sum of endomorphisms $\eta \mapsto \zeta\left\langle\zeta^{\prime}, \eta\right\rangle$ with $\zeta, \zeta^{\prime} \in Y_{A}$ ), together with the fact that, by definition, $t_{A}$ is a limit in norm of endomorphisms of finite rank (and, also, the fact that the push-forward of an arbitrary adjointable endomorphism is seen by the calculation outlined above to have at most the same norm).

This shows that the correspondence $X_{A} \rightarrow X_{B}$ is functorial, for a fixed map $A \rightarrow B$, in a way that passes naturally to a morphism $\mathcal{C} u(A) \rightarrow \mathcal{C} u(B)$.

Let us now show that the resulting functor, from the category of all $\mathrm{C}^{*}$-algebras to the category $\mathcal{C} u$ - for it is manifestly a functor (i.e., respects composition of maps) - , preserves (sequential) inductive limits.

Let $A_{1} \rightarrow A_{2} \rightarrow \cdots$ be a sequence of $\mathrm{C}^{*}$-algebras, with inductive limit $A$. Let us show that if $X$ is a countably generated Hilbert $\mathrm{C}^{*}$-module over $A$, then the class $[X]$ of $X$ in the Cuntz semigroup of $A$ is the supremum of the increasing sequence consisting of the canonical images in this semigroup of a sequence $\left(x_{i}\right)$ with $x_{i}$ in the Cuntz semigroup of $A_{i}$ and with $x_{i} \leq x_{i+1}$ for each $i$, where $x_{i}$ denotes the image of $x_{i}$ in the Cuntz semigroup of $A_{i+1}$ and the comparison is in that ordered semigroup. As we shall show below, this makes it possible to deduce, just from the 
construction of the inductive limit of a sequence in the category $\mathcal{C} u$ given above at the beginning of the proof, that the Cuntz semigroup of $A$-let us denote this by $\mathcal{C} u(A)$ - is the inductive limit (in $\mathcal{C} u$ ) of the sequence $\mathcal{C} u\left(A_{1}\right) \rightarrow \mathcal{C} u\left(A_{2}\right) \rightarrow \cdots$, corresponding to the given sequence $A_{1} \rightarrow A_{2} \rightarrow \cdots$.

By Theorem 2 of [14], $X$ is isomorphic to a subobject of the countably infinite Hilbert $\mathrm{C}^{*}$-module direct sum, $\bigoplus_{1}^{\infty} A$, of copies of $A$, and so we may suppose that it is a subobject of $\bigoplus_{1}^{\infty} A$. Although $A$ itself may not be countably generated as a Hilbert $A$-module, and so also not $\bigoplus_{1}^{\infty} A$, there exists a countably generated closed submodule $A^{\prime}$ of $A$ such that $\bigoplus_{1}^{\infty} A^{\prime}$ contains $X$ - for instance, that generated by all the coordinates in $\bigoplus_{1}^{\infty} A$ of a countable generating set for $X \subseteq \bigoplus_{1}^{\infty} A$. Denote $\bigoplus_{1}^{\infty} A^{\prime}$ by $Y$.

Concerning the object $Y$, we shall use only that $Y$ contains $X$, and that there exists a sequence $Y_{1} \subseteq Y_{2} \subseteq \cdots \subseteq Y$ of subobjects of $Y$ such that each $Y_{n}$ arises from some finite stage of the sequence $A_{1} \rightarrow A_{2} \rightarrow \cdots \rightarrow A$, by the functorial pushforward construction described above. Passing to a subsequence of $A_{1} \rightarrow A_{2} \rightarrow \cdots$ we may suppose that $Y_{n}$ arises form the $n$th stage, say from the object $\left(Y_{n}\right)_{A_{n}}$ over $A_{n}$. (Let us then sometimes write $Y_{n}$ for $\left(Y_{n}\right)_{A_{n}}$, and $\left(Y_{n}\right)_{A}$ for the push-forward!) (To obtain the desired increasing sequence $Y_{1} \subseteq Y_{2} \subseteq \ldots$ of subobjects of $Y$ over $A$, use that $Y=\bigoplus_{1}^{\infty} A^{\prime}$, and, if the closed right $A$-module $A^{\prime}$ is not already the closure of the union of the push-forwards $\left(A^{\prime} \cap A_{1}\right) A,\left(A^{\prime} \cap A_{2}\right) A, \cdots$ of $A_{1}, A_{2}, \cdots$ respectively, then simply enlarge $A^{\prime}$ (by adjoining countably many elements, of $A_{1}, A_{2}, \cdots$ approximating the generators of $A^{\prime}$ ), so that this is the case.) It follows in particular that every compact endomorphism of $Y$ is the limit of a sequence of compact endomorphisms arising from $\left(Y_{n}\right)_{A_{n}}$, for $=1,2, \cdots$.

Let us now construct as promised an increasing sequence $x_{1} \leq x_{2} \leq \cdots$ in $\mathcal{C} u(A)$, with $x_{i}$ arising from an element of $\mathcal{C} u\left(A_{i}\right)$ for each $i$, with $x_{i} \leq x_{i+1}$ in $\mathcal{C} u\left(A_{i+1}\right)$ for each $i$, and with $\sup x_{i}=[X]$. Choose a strictly positive element, $h$, of the compact endomorphism algebra of $X$, and recall that then $h$ belongs in a natural way to the compact endomorphism algebra of $Y$, of which $X$ is assumed to be a subobject. Recall that $Y=\left(\bigcup\left(Y_{i}\right)_{A}\right)^{-}$where $Y_{i}$ is a Hilbert $A_{i}$-module for each $i$ with $\left(Y_{i}\right)_{A_{i+1}} \subseteq Y_{i+1}$, so that in particular $\left(Y_{1}\right)_{A} \subseteq\left(Y_{2}\right)_{A} \subseteq \cdots$, and that, furthermore, every compact endomorphism of $Y$ can be approximated arbitrarily closely in norm by the push-forwards of compact endomorphisms of $Y_{1}, Y_{2}, \cdots$. Choose a compact endomorphism $h_{n} \geq 0$ of $Y_{n}$ such that $h_{n}=\left(h_{n}\right)_{A}$ tends to $h$ in the compact endomorphism algebra of $Y$. By Lemma 2.2 of [17], for given $\epsilon>0$, choosing $h_{n}$ close enough to $h$-strictly within $\epsilon$ is sufficient (at this stage!-see 
below) - we obtain

$$
\left(h_{n}-\epsilon\right)_{+}=d h d^{*}
$$

for some compact endomorphism $d$ of $Y$ (which may be chosen to have norm one, but we don't need this). Furthermore, inspection of the construction of $d$ in the proof of Lemma 2.2 of [17] shows that, with $d$ constructed in this way, the element $h^{\frac{1}{2}} d^{*} d h^{\frac{1}{2}}$, a compact endomorphism of $X$, is close (in norm) to $h$ - to obtain this it is no longer sufficient for $h_{n}$ just to be within $\epsilon$ of $h$, but for a given desired degree of approximation by $h^{\frac{1}{2}} d^{*} d h^{\frac{1}{2}}$ - let us say $\epsilon$ ! - we may just choose $h_{n}$ to give the necessary approximation to $h$. Then, again by Lemma 2.2 of [17],

$$
(h-\epsilon)_{+}=e h^{\frac{1}{2}} d^{*} d h^{\frac{1}{2}} e^{*}
$$

for some compact endomorphism $e$ of $X$. Combining these two equations, we see that $\left((h-\epsilon)_{+} X\right)^{-}$, a (compactly contained) subobject of $X$, is isomorphic to a subobject of $\left(\left(h_{n}-\epsilon\right)_{+} Y\right)^{-}$. (The partially isometric part of the compact homomorphism $d h^{\frac{1}{2}} e^{*}$ is a (not necessarily adjointable) isometry from $\left((h-\epsilon)_{+} X\right)^{-}$ to $\left(\left(h_{n}-\epsilon\right)_{+} Y\right)^{-}$, with image $\left(d h^{\frac{1}{2}} e^{*} e h^{\frac{1}{2}} d^{*} Y\right)^{-} \subseteq\left(\left(h_{n}-\epsilon\right)_{+} Y\right)^{-}$.) Therefore, in $\mathcal{C} u(A)$,

$$
\left[\left((h-\epsilon)_{+} X\right)^{-}\right] \leq\left[\left(\left(h_{n}-\epsilon\right)_{+} Y\right)^{-}\right]
$$

The important point is that the object $\left(\left(h_{n}-\epsilon\right)_{+} Y\right)^{-}$is the closure of the union of the increasing sequence of subobjects $\left(\left(h_{n}-\epsilon\right)_{+}\left(Y_{k}\right)_{A}\right)^{-}$, each one of which arises from a finite stage - namely, $\left(\left(h_{n}-\epsilon\right)_{+}\left(Y_{k}\right)_{A}\right)^{-}$is the push-forward of $\left(\left(h_{n}-\epsilon\right)_{+}\left(Y_{k}\right)_{A_{l}}\right)^{-}$, where $l=\max (k, n)$. As shown earlier, the first property implies that, in $\mathcal{C} u(A)$,

$$
\left[\left(\left(h_{n}-\epsilon\right)_{+} Y\right)^{-}\right]=\sup _{k}\left[\left(\left(h_{n}-\epsilon\right)_{+}\left(Y_{k}\right)_{A}\right)^{-}\right] .
$$

At the same time, on choosing a sequence $\epsilon_{m}$ tending (strictly) monotonically to 0 , one has

$$
X=\left(\bigcup\left(\left(h-\epsilon_{m}\right)_{+} X\right)^{-}\right)^{-},
$$

and hence, in $\mathcal{C} u(A)$,

$$
[X]=\sup _{m}\left[\left(\left(h-\epsilon_{m}\right)_{+} X\right)^{-}\right] .
$$

Finally, note that, also, for each $\epsilon$, with $h_{n}$ as above, the object $\left(\left(h_{n}-\epsilon\right)_{+} Y\right)^{-}$ is isomorphic to a subobject of $X$, by means of the partially isometric part of the compact homomorphism $h^{\frac{1}{2}} d^{*}$, from $\left(\left(h_{n}-\epsilon\right)_{+} Y\right)^{-}$to $X=(h Y)^{-}$. Making $\epsilon$ 
smaller, we may ensure that the image is compactly contained in $X$. Hence, as shown earlier,

$$
\left[\left(\left(h_{n}-\epsilon\right)_{+} Y\right)^{-}\right]<<[X]
$$

in $\mathcal{C} u(A)$. The conclusion now follows, with $x_{1}, x_{2}, \cdots$ chosen after passing to the subsequence $A_{k_{1}} \rightarrow A_{k_{2}} \rightarrow \cdots$ of $A_{1} \rightarrow A_{2} \rightarrow \cdots$ to be the sequence (with respect to the given sequence $A_{1} \rightarrow A_{2} \rightarrow \cdots$ )

$$
y_{m}^{\prime}=\left[\left(\left(h_{n_{m}}-\epsilon_{l_{m}}\right)_{+}\left(Y_{k_{m}}\right)_{A}\right)^{-}\right], m=1,2, \cdots
$$

(arising as observed above from finite stages), for suitable sequences $\left(n_{m}\right),\left(l_{m}\right)$ and $\left(k_{m}\right)$. Indeed, first choose $n_{1}$ such that

$$
\left[\left(\left(h-\epsilon_{2}\right)_{+} X\right)^{-}\right] \leq\left[\left(\left(h_{n_{1}}-\epsilon_{2}\right)_{+} Y\right)^{-}\right]
$$

Since $\epsilon_{2}<\epsilon_{1}$, we have $\left(\left(h_{n_{1}}-\epsilon_{2}\right)_{+} Y\right)^{-} \subset \subset\left(\left(h_{n_{1}}-\epsilon_{1}\right)_{+} Y\right)^{-}$, whence by the ordertheoretic compact inclusion of the corresponding Cuntz classes in $\mathcal{C} u(A)$, we may choose $k_{1}$ such that

$$
\left[\left(\left(h_{n_{1}}-\epsilon_{2}\right)_{+} Y\right)^{-}\right] \leq\left[\left(\left(h_{n_{1}}-\epsilon_{1}\right)_{+} Y_{k_{1}}\right)^{-}\right]
$$

By compact inclusion of the latter class in $[X]$, we may choose $l_{2}$ such that

$$
\left[\left(\left(h_{n_{1}}-\epsilon_{1}\right)_{+} Y_{k_{1}}\right)^{-}\right] \leq\left[\left(\left(h-\epsilon_{l_{2}+1}\right)_{+} X\right)^{-}\right]
$$

Choose $n_{2}$ in the same way as $n_{1}$ above such that

$$
\left[\left(\left(h-\epsilon_{l_{2}+1}\right)_{+} X\right)^{-}\right] \leq\left[\left(\left(h_{n_{2}}-\epsilon_{l_{2}+1}\right)_{+} Y\right)^{-}\right] .
$$

Again by compactness, as $\epsilon_{l_{2}+1}<\epsilon_{l_{2}}$, we may choose $k_{2}$ in the same way as $k_{1}$ above such that

$$
\left[\left(\left(h_{n_{2}}-\epsilon_{l_{2}+1}\right)_{+} Y\right)^{-}\right] \leq\left[\left(\left(h_{n_{2}}-\epsilon_{l_{2}}\right)_{+} Y_{k_{2}}\right)^{-}\right]
$$

Continuing in the way just described, we obtain an increasing sequence $y_{m}^{\prime}=$ $\left[\left(\left(h_{n_{m}}-\epsilon_{l_{m}}\right)_{+}\left(Y_{k_{m}}\right)_{A}\right)^{-}\right]$in $\mathcal{C} u(A)$ which is intertwined with respect to the order relation with the increasing sequence $x_{m}^{\prime}=\left[\left(\left(h-\epsilon_{l_{m}+1}\right)_{+} X\right)^{-}\right]$- and is in particular increasing! Since the second sequence, $\left(x_{m}^{\prime}\right)$, as shown earlier, has supremum $[X]$ in $\mathcal{C} u(A)$, the first sequence, $\left(y_{m}^{\prime}\right)$, also does. It remains to note that after passing to the subsequence $A_{k_{1}} \rightarrow A_{k_{2}} \rightarrow \cdots$ and changing notation, $y_{m}^{\prime}$ belongs 
to $\mathcal{C} u\left(A_{m}\right)$ for each $m$, and so the sequence $\left(y_{m}^{\prime}\right)$ fulfils the requirements for the sequence $\left(x_{m}\right)$ - except possibly for the condition $y_{m}^{\prime} \leq y_{m+1}^{\prime}$ in $\mathcal{C} u\left(A_{m+1}\right)$. This is ensured by again applying Lemma 2.2 of [17], to refine the choice of the sequences $\left(n_{m}\right),\left(k_{m}\right)$, and $\left(l_{m}\right)$ so that, for each $m$,

$$
\left(h_{n_{m}}-\epsilon_{l_{m}}\right)_{+}=d\left(h_{n_{m+1}}-\epsilon_{l_{m+1}}\right)_{+} d^{*}
$$

for some compact endomorphism $d$ of $Y_{n_{m+1}} \subseteq Y_{k_{m+1}}$, from which it follows that $\left(\left(h_{n_{m}}-\epsilon_{l_{m}}\right)_{+} Y_{k_{m}}\right)^{-}$is isomorphic to a subobject of $\left(\left(h_{n_{m+1}}-\epsilon_{l_{m+1}}\right)_{+} Y_{k_{m+1}}\right)^{-}$, over $A_{k_{m+1}}$-then one has $y_{m}^{\prime} \leq y_{m+1}^{\prime}$ in $\mathcal{C} u\left(A_{k_{m+1}}\right)$, or in $\mathcal{C} u\left(A_{m+1}\right)$ after the prescribed passage to the subsequence $A_{k_{1}} \rightarrow A_{k_{2}} \rightarrow \cdots$ of $A_{1} \rightarrow A_{2} \rightarrow \cdots$, as desired.

Let us now show that, with respect to the canonical sequence in the category $\mathcal{C} u$ corresponding to the sequence of $\mathrm{C}^{*}$-algebras $A_{1} \rightarrow A_{2} \rightarrow \cdots \rightarrow A$,

$$
\mathcal{C} u\left(A_{1}\right) \rightarrow \mathcal{C} u\left(A_{2}\right) \rightarrow \cdots \rightarrow \mathcal{C} u(A)
$$

$\mathcal{C} u(A)$ is the inductive limit. By the construction of $\lim \mathcal{C} u\left(A_{i}\right)$ in the proof of Theorem 2, and what has just been proved, it is sufficient to show that if $x_{1} \leq x_{2} \leq$ $\cdots$ with $x_{i} \in \mathcal{C} u\left(A_{i}\right)$ and also $y_{1} \leq y_{2} \leq \cdots$ with $y_{i} \in \mathcal{C} u\left(A_{i}\right)$, then $\sup x_{i} \leq \sup y_{i}$ in $\mathcal{C} u(A)$ (the suprema of course referring to the images of the sequences $\left(x_{i}\right)$ and $\left(y_{i}\right)$ in $\left.\mathcal{C} u(A)\right)$ if, and only if, whenever $z<<x_{i}$ in $\mathcal{C} u\left(A_{i}\right)$ for some $i$ then $z<<y_{j}$ in $\mathcal{C} u\left(A_{j}\right)$ for some $j \geq i$. (This establishes an isomorphism of ordered semigroups from $\lim _{\rightarrow} \mathcal{C} u\left(A_{i}\right)$ onto a sub ordered semigroup of $\mathcal{C} u(A)$, and this subsemigroup was shown above to be all of $\mathcal{C} u(A)$.)

Replacing $\left(x_{i}\right)$ and $\left(y_{i}\right)$ with equivalent sequences (which does not change the statement of what is to be proved), as in the proof of Theorem 1, we may suppose, first, that $\left(x_{i}\right)$ and $\left(y_{i}\right)$ are rapidly increasing, and, second, that $x_{i}=\left[X_{i}\right]$ and $y_{i}=\left[Y_{i}\right]$ where $X_{i}$ and $Y_{i}$ are Hilbert $\mathrm{C}^{*}$-modules over $A_{i}$ and

$$
X_{1} \subset \subset X_{2} \subset \subset \cdots, \quad Y_{1} \subset \subset Y_{2} \subset \subset \cdots
$$

Then

$$
\sup x_{i}=\left[\lim _{\rightarrow}\left(X_{i}\right)_{A}\right] \text { and } \sup y_{i}=\left[\lim _{\rightarrow}\left(Y_{i}\right)_{A}\right]
$$

where $\left(X_{i}\right)_{A}$ denotes the push-forward of $X_{i}$ from $A_{i}$ to $A$, discussed above.

Suppose first that $\sup x_{i} \leq \sup y_{i}(\operatorname{in} \mathcal{C} u(A))$, and let $z<<x_{i}$ in $\mathcal{C} u\left(A_{i}\right)$ be given for some fixed $i$. By the concrete definition of $<<$ (see proof of Theorem 1 ),

$$
z \leq\left[Z^{\prime}\right] \text { for some } Z^{\prime} \subset \subset X_{i} \text { over } A_{i} \text {. }
$$


Furthermore, we may choose $Z^{\prime \prime}$ and $Z^{\prime \prime \prime}$ such that

$$
Z^{\prime} \subset \subset Z^{\prime \prime} \subset \subset Z^{\prime \prime \prime} \subset \subset X_{i} \text { over } A_{i}
$$

Then,

$$
Z_{A}^{\prime} \subset \subset Z_{A}^{\prime \prime} \subset \subset Z_{A}^{\prime \prime \prime} \subset \subset\left(X_{i}\right)_{A}
$$

In particular, $\left[Z^{\prime \prime \prime}\right]<<\left[X_{i}\right] \leq \sup x_{i} \leq \sup y_{i}($ in $\mathcal{C} u(A))$, and so by the abstract definition of $<<$,

$$
\left[Z^{\prime \prime \prime}\right] \leq\left[Y_{j}\right] \text { for some } j \text {, in } \mathcal{C} u(A) \text {. }
$$

It follows, in particular (by definition) that $Z^{\prime \prime}$ is isomorphic to a compactly contained subobject of $Y_{j}$ over $A$, say $Y_{j}^{\prime}$. Let $h$ be a positive element of the algebra of compact endomorphisms of $\left(Z^{\prime \prime}\right)_{A_{i}}$ (to be specified later!). This is then $x^{*} x$ where $x x^{*}$ is a compact endomorphism of $\left(Y_{j}\right)_{A}$, for a certain compact homomorphism $x$ from $\left(Z^{\prime \prime}\right)_{A}$ to $\left(Y_{j}\right)_{A}$ (namely, the product of $\left(h^{\frac{1}{2}}\right)_{A}$ with an isomorphism from $\left(Z_{A_{i}}^{\prime \prime}\right)_{A}$ to a subobject of $\left.\left(Y_{j}\right)_{A}\right)$. Since $Z^{\prime \prime}$ and $Y_{j}$ arise from the $i$ th and $j$ th stages, respectively, for some $k \geq \max (i, j)$ we may approximate $x$ in norm, in the algebra of compact homomorphisms from $\left(Z^{\prime \prime}\right)_{A}$ to $\left(Y_{j}\right)_{A}$, by a compact homomorphism from $\left(\left(Z^{\prime \prime}\right)_{A_{i}}\right)_{A_{k}}$ to $\left(\left(Y_{j}\right)_{A_{j}}\right)_{A_{k}}$, say $x^{\prime}$. Then $x^{\prime} * x^{\prime}$ is close to $h$ in the algebra of compact endomorphisms of $\left(Z_{A_{i}}^{\prime \prime}\right)_{A}$, and since both $h$ and $x^{\prime} * x^{\prime}$ arise from a finite stage, say for the moment $\left(Z_{A_{i}}^{\prime \prime}\right)_{A_{k}}$ for fixed $k$, if they are close over $A$ then they are also (almost as) close over $A_{l}$ for some $l \geq k$. Hence by Lemma 2.2 of [17], correcting $x^{\prime}$ by composing with a compact endomorphism of $\left(\left(Z^{\prime \prime}\right)_{A_{i}}\right)_{A_{l}}$, we may suppose that $x^{\prime} * x^{\prime}=(h-\epsilon)_{+}$, for a given $\epsilon>0$-if we choose $k$ large to begin with (and then $l$ large depending on this choice).

Now let us use that, since $Z^{\prime} \subset \subset Z^{\prime \prime}$ over $A_{i}$, there exists a compact self-adjoint endomorphism $h=h_{A_{i}}$ of $\left(Z^{\prime \prime}\right)_{A_{i}}$ equal to the identity on $Z^{\prime}$. As shown earlier, we may suppose not only that $h$ is positive, but that also in fact $(h-\epsilon)+$ acts as the identity on $Z^{\prime} \subset Z^{\prime \prime}$. This gives the choice of $h$ and $\epsilon$ to be used above. We thus obtain that $x^{\prime}$ is a compact homomorphism from $\left(Z^{\prime \prime}\right)_{A_{l}}$ to $\left(Y_{j}\right)_{A_{l}}$ such that $x^{\prime} * x^{\prime}=$ $(h-\epsilon)_{+}$, from which we deduce that the restriction of $x^{\prime}$ to $\left(Z^{\prime}\right)_{A_{l}} \subset \subset\left(Z^{\prime \prime}\right)_{A_{l}}$ is an isomorphism from $\left(Z^{\prime}\right)_{A_{l}}$ to a compactly contained subobject of $\left(Y_{j}\right)_{A_{l}}$ (as $x^{\prime} x^{\prime} *$ is a compact endomorphism of $\left(Y_{j}\right)_{A_{l}}$ acting as the identity on the image of $\left(Z^{\prime}\right)_{A_{l}}$ by $x^{\prime}$ - given that the compact self-adjoint endomorphism $x^{\prime} * x^{\prime}\left(=(h-\epsilon)_{+}\right)$of $\left(Z^{\prime \prime}\right)_{A_{l}}$ acts as the identity on $\left.\left(Z^{\prime}\right)_{A_{l}}\right)$, as desired.

Suppose, conversely, that whenever $z<<x_{i}$ in $\mathcal{C} u\left(A_{i}\right)$ for some $i$ then $z<<y_{j}$ in $\mathcal{C} u\left(A_{j}\right)$ for some $j \geq i$, and let us show that $\sup x_{i} \leq \sup y_{i}$ in $\mathcal{C} u(A)$. We 
must show that $x_{i} \leq \sup y_{j}$ in $\mathcal{C} u(A)$ for every $i$. Let us then fix $i=1,2, \cdots$. By Theorem $1, x_{i}$ is the supremum in $\mathcal{C} u\left(A_{i}\right)$ of an increasing sequence $\left(z_{n}\right)$ in $\mathcal{C} u\left(A_{i}\right)$ with $z_{n}<<x_{i}$ in $\mathcal{C} u\left(A_{i}\right)$ for each $n=1,2, \cdots$. Then by hypothesis, for each $n$ we have $z_{n}<<y_{j}$ in $\mathcal{C} u\left(A_{j}\right)$ for some $j \geq i$. Then also (by functoriality) $z_{n}<<y_{j}$ in $\mathcal{C} u(A)$ - where now $j$ is fixed but $n$ is arbitrary. In fact, all we shall need is $z_{n} \leq y_{j}$. By functoriality, from $x_{i}=\sup z_{n}$ in $\mathcal{C} u\left(A_{i}\right)$ follows $x_{i}=\sup z_{n}$ in $\mathcal{C} u(A)$, and so $x_{i}=\sup z_{n} \leq y_{j}$ in $\mathcal{C} u(A)$, as desired.

3. The following result is a partial answer to the questions concerning Cuntz equivalence raised in Section 1.

Theorem. Let $A$ be a $C^{*}$-algebra of stable rank one. Two countably generated Hilbert $C^{*}$-modules over $A$ are equivalent in the sense of Cuntz (described in Section 1) if, and only if, they are isomorphic. In other words, the Cuntz semigroup in this case is just the semigroup of isomorphism classes of countably generated Hilbert $C^{*}$-modules. Furthermore, the order structure arises from inclusion of modules.

Proof. Since isomorphic Hilbert $\mathrm{C}^{*}$-modules have the same isomorphism classes of compactly contained subobjects, by definition they are Cuntz equivalent.

Let $X$ and $Y$ be Cuntz equivalent countably generated Hilbert $\mathrm{C}^{*}$-modules over $A$, and let us show that $X$ and $Y$ are isomorphic. Choose (as described above) rapidly increasing sequences of subobjects

$$
\begin{aligned}
& X_{1} \subset \subset X_{1} \subset \subset \cdots \subseteq X \\
& Y_{1} \subset \subset Y_{2} \subset \subset \cdots \subseteq Y,
\end{aligned}
$$

generating $X$ and $Y$ respectively. By the definition of Cuntz equivalence, $X_{2}$ is isomorphic to a compactly contained subobject of $Y$. In particular (note that this is a priori a weaker property), $\left[X_{2}\right] \subset \subset[Y]$ (i.e., $\left[X_{2}\right] \leq[Z]$ for some $Z \subset \subset Y$ ), and hence as shown in the proof of Theorem $1,\left[X_{2}\right]<<[Y]$. As shown in the proof of Theorem 2, $[Y]=\sup \left[Y_{i}\right]$. It follows from the definition of compact containment (of Cuntz equivalence classes) in the order-theoretic sense that $\left[X_{2}\right] \leq\left[Y_{i}\right]$ for some $i=1,2, \cdots$. In particular, on choosing $i_{1}$ with $\left[X_{2}\right] \leq\left[Y_{i_{1}}\right]$, we have by definition that, as $X_{1} \subset \subset X_{2}$, the object $X_{1}$ is isomorphic to a subobject of $Y_{i_{1}}$, say by the $\operatorname{map} \varphi_{1}: X_{1} \rightarrow Y_{i_{1}}$.

In a similar way (considering first $Y_{i_{1}+1} \subset \subset Y$ ) we obtain an isomorphism $\psi_{1}$ of $Y_{i_{1}}$ onto a subject of $X_{j_{1}}$ for some $j_{1}=1,2, \cdots$. Continuing in this way, and 
passing to subsequences of $\left(X_{i}\right)$ and $\left(Y_{i}\right)$ and changing notation, we have a diagram

$$
\begin{gathered}
X_{1} \subset \subset \quad X_{2} \quad \subset \subset \cdots \subseteq X \\
\varphi_{1} \downarrow \psi_{1} \nearrow \varphi_{2} \downarrow \psi_{2} \nearrow \\
Y_{1} \subset \subset \quad Y_{2} \quad \subset \subset \cdots \subseteq Y,
\end{gathered}
$$

in which each vertical map (downwards or upwards) is an isomorphism onto its image.

It is sufficient, by a modification of 2.2 and 2.1 of [6] (using that $X_{i}$ and $Y_{i}$ are countably generated) - see Example 4.4 and Theorem 3 of [7] - to show that any two isomorphisms from one Hilbert $\mathrm{C}^{*}$-module over A onto submodules of another one are approximately equal, on finitely many elements, modulo inner automorphisms of the codomain Hilbert module, i.e., automorphisms arising from unitary elements of the $\mathrm{C}^{*}$-algebra of compact endomorphisms with the identity adjoined. (In other words, any two such homomorphisms are close on finitely many elements after one of them is composed with such an automorphism.)

Let us establish this fact, using, naturally, that $A$ has stable rank one. It is enough to show that, in this case, an isomorphism between two closed submodules of a Hilbert $\mathrm{C}^{*}$-module can be approximated pointwise by an inner automorphism (defined as above) of the whole module. The first step is to note that, by Proposition 1.3 of [15], such an isomorphism can be approximated on each finite set by a compact homomorphism of norm one and that - cf. above - such a homomorphism extends to a compact endomorphism of norm one of the larger Hilbert module. The second step is to note that, as the property of having stable rank one is invariant under RieffelMorita equivalence, the $\mathrm{C}^{*}$-algebra of compact endomorphisms of the given Hilbert $\mathrm{C}^{*}$-module has stable rank one - and so each element of the algebra of compact endomorphisms with unit adjoined can be approximated in norm by an invertible element of this $\mathrm{C}^{*}$-algebra - of norm one if the given element is of norm one. The final step is to note that, if an element of the algebra of compact endomorphisms of a Hilbert $\mathrm{C}^{*}$-module with unit adjoined (or even just an adjointable endomorphism) approximately preserves inner products on a given finite subset, and in addition has norm one, then its absolute value is close to the identity on these elements - as, for an adjointable endomorphism $x$ of norm at most one and a Hilbert $\mathrm{C}^{*}$-module element $\xi$,

$$
\begin{aligned}
\langle(1-|x|) \xi,(1-|x|) \xi\rangle & =\left\langle\xi,(1-|x|)^{2} \xi\right\rangle \\
& \leq\langle\xi,(1-|x|)(1+|x|) \xi\rangle \\
& =\left\langle\xi,\left(1-x^{*} x\right) \xi\right\rangle \\
& =\langle\xi, \xi\rangle-\langle x \xi, x \xi\rangle
\end{aligned}
$$


- and so if in addition this endomorphism is invertible then it is close, on the given finite subset, to its unitary part - a unitary element of the $\mathrm{C}^{*}$-algebra of compact endomorphisms with unit adjoined, and so an inner automorphism as defined above.

Finally, let $X$ and $Y$ be countably generated Hilbert $\mathrm{C}^{*}$-modules such that $[X] \leq$ $[Y]$, and let us show that $X$ is isomorphic to a subobject of $Y$. One has a diagram as above but without the upwards arrows. As above, the downwards arrows may be modified one by one by composing with inner automorphisms in such a way as to ensure that each square is arbitrarily close to commuting on an arbitrary finite set. As in 2.2 of [6], these finite sets may be chosen in such a way that the diagram is approximately commutative in the sense of 2.1 of [6], and hence by 2.1 of [6] there is a (unique) contraction from $X$ to $Y$ such that the diagram remains commutative - and this map is easily seen to be an isomorphism of $X$ onto a subobject of $Y$.

4. The following consequence of Theorems 1, 2, and 3 (and in particular of Lemma 2.2 of [17]) is of interest from the point of view of the classification of $\mathrm{C}^{*}$ algebras. (By [9], it implies that arbitrary simple $\mathrm{C}^{*}$-algebras stably isomorphic to a separable simple AI algebra are classified by their Elliott invariant.)

Corollary. Let $A$ be the inductive limit of a sequence of $C^{*}$-algebras $A_{1} \rightarrow$ $A_{2} \rightarrow \cdots$, and let $B$ be a hereditary sub- $C^{*}$-algebra of $A$. It follows that for every finite subset of $B$ there is a sub- $C^{*}$-algebra of $B$ approximately containing this finite subset and isomorphic to the image in $A$ of a hereditary sub- $C^{*}$-algebra of $A_{n}$ for some $n$. Hence in particular, if $A$ is separable and if either $A$ has stable rank one, or quotients of hereditary sub-C $C^{*}$-algebras of $A_{n}$ are weakly semiprojective for each $n=1,2, \cdots$, then $B$ is isomorphic to the inductive limit of a sequence of such $C^{*}$-algebras.

Proof. The proof of the first assertion is very similar to part of the proof of Theorem 2, above, but is self-contained - in the sense that it appeals directly (in the same way as above) to Lemma 2.2 of [17] and its proof.

Given a finite subset $F$ of $B$, choose a positive element $h$ of $B$ of norm one which is close to the identity on $F$, acting in either side, and choose a positive element $h_{m}$ of the image of $A_{m}$ in $A$ for some $n$ that is close to $h$, say to strictly within distance $\epsilon$. Then by Lemma 2.2 of [17],

$$
\left(h_{n}-\epsilon\right)_{+}=d h d^{*}
$$

for some $d \in A$ of norm at most one. (Here we need the contraction propertywhen this theorem is used earlier we could just as well have used Lemma 2.5(ii) of 
[16] in which the contraction property is not assured.) Furthermore, inspection of the construction of $d$ in [17] shows that, if $h_{m}$ is sufficiently close to $h$, say to within distance $\epsilon^{\prime}$ (where $\epsilon^{\prime} \leq \epsilon$ ), then the element $h^{\frac{1}{2}} d^{*} d h^{\frac{1}{2}}$ of $A$ is also close to $h$. Then with $a=d h^{\frac{1}{2}}$, the hereditary sub-C*-algebras of $A$ generated by $a^{*} a=h^{\frac{1}{2}} d^{*} d h^{\frac{1}{2}}$ and $a a^{*}=d h d^{*}=\left(h_{m}-\epsilon\right)_{+}$are isomorphic. The first, that generated by $a^{*} a$, is contained in $B$ and contains the finite set $a^{*} a F a^{*} a$. Since $a^{*} a$ is close to $h$ and is of norm at most one (as $h$ and $d$ are), so that $a^{*} a F a^{*} a$ is close to $h F h$, and since $h F h$ is close to $F$ (as $h$ is close to the identity on $F$ on either side and has norm one), the finite set $a^{*} a F a^{*} a$ is close to $F$. The hereditary sub-C*-algebra of $A$ generated by $a^{*} a$ thus is contained in $B$ and approximately contains $F$. The second hereditary sub-C*-algebra, that generated by $a a^{*}$, is isomorphic to this, and, since $a^{*} a=\left(h_{n}-\epsilon\right)_{+}$, for $m$ sufficiently large the pre-image of the finite subset of the first algebra approximating $F$ is approximated by the closure of $\left(h_{m}-\epsilon\right)_{+} A_{n}\left(h_{m}-\epsilon\right)_{+}$, where $A_{n}$ denotes also the image of $A_{n}$ in $A$. This closure is then the image in $A$ of a hereditary sub-C*-algebra of $A_{n}$, and, as a subalgebra (although not a hereditary subalgebra) of $A$ is isomorphic to a sub- $C^{*}$-algebra of $B$ approximately containing $F$, as desired.

Consider now the second assertion of the theorem.

If the sub- $C^{*}$-algebras of $B$ constructed in the way described above are weakly semiprojective, as they are if all quotients of hereditary sub- $\mathrm{C}^{*}$-algebras of $A_{1}, A_{2}, \cdots$ are assumed to be weakly semiprojective, and if $B$ is separable, then an iterative construction using weak semiprojectivity together with an interwining argument yields an increasing sequence of such subalgebras with union dense in $B$, as desired.

An outline of this construction follows. (The proof is slightly indirect.) Choose first a sequence $D_{1}, D_{2}, \cdots$ of such subalgebras such that for each $i$, a suitably large subset of $D_{i}$ (to be specified) is approximately contained in $D_{i+1}$. Then, by weak semiprojectivity (maps from $D_{i}$ into the asymptotic sequence algebra $\Pi_{n=i}^{\infty} D_{n} / \bigoplus_{n=i}^{\infty} D_{n}$ lift), we obtain, after passing to a subsequence of $D_{1}, D_{2}, \cdots$, a sequence of $\mathrm{C}^{*}$-algebra maps $D_{1} \rightarrow D_{2} \rightarrow \cdots$ such that the diagram

$$
\begin{aligned}
& D_{1} \rightarrow D_{2} \rightarrow \cdots \\
& \downarrow \quad \downarrow \\
& B \rightarrow B \rightarrow \cdots
\end{aligned}
$$


approximately commutes, in the sense of 2.2 and 2.1 of [6], where the maps $B \rightarrow$ $B$ are the identity and the vertical maps are the inclusions. By 2.1 of [6], the diagram extends to an approximately commutative diagram including a (unique) map $\lim _{\rightarrow} D_{i} \rightarrow B=\lim _{\rightarrow} B$, and if the subalgebras $D_{1}, D_{2}, \cdots$ of $B$ are chosen to approximate a dense sequence of elements of $B$ in a suitable way (finitely many at each stage, eventually more and more, better and better), then the map $\lim _{\rightarrow} D_{i} \rightarrow B$ is surjective, as desired. (Cf. [23].)

Now suppose that $A$ has stable rank one. In this case, we can still prove the second assertion of the theorem, but only by applying the full force of Theorems 1 , 2, and 3. (Not just as a consequence of the first assertion - at least not as simple a one as in the case considered above - the intertwining argument for which was already somewhat indirect!) We, in fact also need to use the proof of Theorem 2, not just the statement - and not just the description of inductive limits in the category $\mathcal{C} u$ and how the functor from the category of $\mathrm{C}^{*}$-algebras to $\mathcal{C} u$ acts on maps, but in fact the detailed construction of how an element $x$ of $\mathcal{C} u(A)$ is expressed as the supremum of an increasing sequence

$$
x_{1} \leq x_{2} \leq \cdots
$$

with $x_{i}$ the image of an element of $\mathcal{C} u\left(A_{i}\right)$.

Since, now, $B$ is separable, $B$ is singly generated as a hereditary sub- $\mathrm{C}^{*}$-algebra of $A$. (In fact, for the present case of the second assertion - that $A$ has stable rank one-it is sufficient only to assume that $B$ is singly generated - not necessarily separable as a $\mathrm{C}^{*}$-algebra.)

The closed right ideal generated by $B$ is then a countably (in fact singly) generated right Hilbert $A$-module; denote this by $X$. By Theorem 2, together with the concrete description of the inductive limit in the category $\mathcal{C} u$ of the sequence $\mathcal{C} u\left(A_{1}\right) \rightarrow \mathcal{C} u\left(A_{2}\right) \rightarrow \cdots$ corresponding to the sequence of $\mathrm{C}^{*}$-algebras $A_{1} \rightarrow A_{2} \rightarrow \cdots$, given in the proof of Theorem 2 , there exists an increasing sequence $x_{1} \leq x_{2} \leq \cdots$ in $\mathcal{C} u(A)$ with $[X]=\sup x_{i}$, such that, for each $i$, the element $x_{i}$ arises from a Hilbert $A_{i}$-module, say $X_{i}$ (by means of the natural map from $\mathcal{C} u\left(A_{i}\right)$ to $\left.\mathcal{C} u(A)\right)$, and such that, moreover, $\left[X_{i}\right] \leq\left[X_{i+1}\right]$ in $\mathcal{C} u\left(A_{i+1}\right)$ for each $i$. In fact, as inspection of the construction in the proof of Theorem 2 shows, the Hilbert $\mathrm{C}^{*}$-modules $X_{1}, X_{2}, \cdots$ over, respectively, $A_{1}, A_{2}, \cdots$ may be chosen such that the push-forward $\left(X_{i}\right)_{A_{i+1}}$ is isomorphic to a subobject of $X_{i+1}$ (over $\left.A_{i+1}\right)$ for each $i$.

Note that, in particular, one has a sequence of Hilbert $A$-module mappings 
(preserving the $A$-valued inner product)

$$
\left(X_{1}\right)_{A} \rightarrow\left(X_{2}\right)_{A} \rightarrow \cdots
$$

and as shown in the proof of Theorem 1 it follows that

$$
\sup x_{i}=\left[\lim _{\rightarrow}\left(X_{i}\right)_{A}\right]
$$

Since $x=[X]$ it follows that

$$
[X]=\left[\lim _{\rightarrow}\left(X_{i}\right)_{A}\right]
$$

Since $A$ has stable rank one, by Theorem 3 we have

$$
X \cong \lim _{\rightarrow}\left(X_{i}\right)_{A}
$$

where the isomorphism is as Hilbert $A$-modules. In particular, the $\mathrm{C}^{*}$-algebra of compact endomorphisms of $X$, i.e., $B$, is isomorphic to the inductive limit of the sequence

$$
B_{1} \rightarrow B_{2} \rightarrow \cdots
$$

where $B_{i}$ denotes the $\mathrm{C}^{*}$-algebra of compact endomorphisms of $\left(X_{i}\right)_{A}$ and $B_{i} \rightarrow$ $B_{i+1}$ the canonical extension map for compact endonorphisms. Now recall that, for each $i$, the Hilbert $A$-module $\left(X_{i}\right)_{A}$ arises from the Hilbert $A_{i}$-module $X_{i}$, and that $\left(X_{i}\right)_{A_{i+1}}$ is isomorphic to a subobject of $X_{i+1}$ (over $A_{i+1}$ ). Note that, for each $i$, the $\mathrm{C}^{*}$-algebra $B_{i}$ of compact endomorphisms of $\left(X_{i}\right)_{A}$ is the inductive limit of the natural sequence of $\mathrm{C}^{*}$-algebras of compact endomorphisms of $\left(X_{i}\right)_{A_{i}}$, $\left(X_{i}\right)_{A_{i+1}}, \cdots$. Let us denote these $\mathrm{C}^{*}$-algebras by $B_{i}^{i}, B_{i}^{i+1}, \cdots$. Thus, for each $i$,

$$
B_{i}=\lim _{\rightarrow} B_{i}^{j}
$$

(where the limit is over $j$ with $j \geq i$ ). The preceding statement implies that, for each $i$, the $\mathrm{C}^{*}$-algebra $B_{i}^{i}$ of compact endomorphisms of $\left(X_{i}\right)_{A_{i}}$ is mapped into the $\mathrm{C}^{*}$ algebra $B_{i+1}^{i+1}$ of compact endomorphisms of $\left(X_{i+1}\right)_{A_{i+1}}$, by the combination of the canonical push-forward to the algebra $B_{i}^{i+1}$ of compact endomorphisms of $\left(X_{i}\right)_{A_{i+1}}$ combined with the canonical map from $B_{i}^{i+1}$ to $B_{i+1}^{i+1}$ corresponding to the canonical extension of compact endomorphisms of $\left(X_{i}\right)_{A_{i+1}}$ to compact endomorphisms of $\left(X_{i+1}\right)_{A_{i+1}}$, with respect to the given isomorphism of $\left(X_{i}\right)_{A_{i+1}}$ with a subobject 
of $\left(X_{i+1}\right)_{A_{i+1}}$. By compatibility of the resulting infinite triangular commutative diagram $\left(B_{i}^{j}\right)_{j \geq i}$ with the horizontal diagram $B_{1} \rightarrow B_{2} \rightarrow \cdots \rightarrow B$ it follows that

$$
B=\lim _{\rightarrow} B_{i}^{i}
$$

in fact as desired since by inspection of the construction each $X_{i}$ has the same number of generators as $X$, namely, one, and so $X_{i}$ is isomorphic to a closed right ideal of $A_{i}$, and so $B_{i}^{i}$ to a hereditary sub-C*-algebra of $A_{i}$.

5. The following consequence of Theorems 1, 2, and 3 is also of interest from the point of view of the classification of $\mathrm{C}^{*}$-algebras. Taken together with the preceding result, it might be viewed as an indication of the potential usefulness of the Cuntz invariant for proving isomorphism of $\mathrm{C}^{*}$-algebras.

Corollary. Let $A$ be a $C^{*}$-algebra of stable rank one. An element of the Cuntz semigroup is compactly contained in itself (in the order-theoretic sense)-let us refer to such an element as compact-if, and only if, it corresponds to a Hilbert $C^{*}$-module which is algebraically finitely generated and projective. (Hence in this case any Cuntz equivalence class also has these properties.)

Furthermore, $A$ is of real rank zero (every hereditary sub-C*-algebra has an approximate unit consisting of projections - see [3]) if, and only if, in the Cuntz semigroup of $A$, every element is the supremum of an increasing sequence of compact elements. Alternatively, an equivalent condition on the Cuntz semigroup is that an element $x$ is compactly contained in an element $y$ (i.e., $x<<y$ ) exactly when $x \leq z \leq y$ for some compact element $z$ (in other words, $x \leq z<<z \leq y$ for some $z$ ). (A different characterization of real rank zero, in the special case that the Cuntz semigroup is almost unperforated, was given in [22].)

In particular, if $A$ is a separable, simple, AH algebra of stable rank one (for instance, by [11], if $A$ has an $A H$ inductive limit decomposition with diagonal maps between building blocks), and if $A$ has the same Cuntz semigroup as a real rank zero AH algebra with an AH inductive limit decomposition with building blocks with spectra of bounded finite dimension, then by [19] and [20] (see also [18]) $A$ is also such an algebra. (Hence by [8], if also $K_{1}(A) \cong K_{1}(B)$ then $A \cong B$.)

Proof. Let $X$ be a Hilbert $\mathrm{C}^{*}$-module over $A$ which is, just considered algebraically as a module, finitely generated and projective. As we shall show below, this is equivalent to the property that $X$ is isomorphic as a (right) Hilbert $A$-module to the submodule of the finite direct sum $A^{\sim} \oplus \cdots \oplus A^{\sim}$ of finitely many copies of $A$ determined by a projection $e$ in the compact endomorphism $\mathrm{C}^{*}$-algebra of the Hilbert $A^{\sim}$-module $A^{\sim} \oplus \cdots \oplus A^{\sim}$-i.e., to the submodule $e\left(A^{\sim} \oplus \cdots \oplus A^{\sim}\right)$, where 
$A^{\sim}=A+\mathbb{C} 1$ is the $\mathrm{C}^{*}$-algebra with unit adjoined, considered as a module over $A$ in the natural way - with the entries of $e$ assumed to belong to $A$, so that the $A^{\sim}$-valued inner product on the submodule determined by $e$ takes values in $A$ so that it is indeed a Hilbert $A$-module. In particular, one sees that the identity endomorphism of $X$ is compact, as the compact endomorphisms of $X$ are just the restrictions to $X$ of the compact endomorphisms of $A^{\sim} \oplus \cdots \oplus A^{\sim}$ taking $X$ into $X$-and these can be identified with the unital algebra $e E e$ where $E$ denotes the algebra of compact endomorphisms of $A^{\sim} \oplus \cdots \oplus A^{\sim}$.

To check the assertion above, note just that, purely algebraically, as an $A$ module, $X$ must be isomorphic to $e\left(A^{\sim} \oplus \cdots \oplus A^{\sim}\right)$ for some idempotent element $e$ of $\mathrm{M}_{n}\left(A^{\sim}\right)$, where $n$ is the number of copies of $A^{\sim}$ in the direct sum (and can be taken to be the number of generators of the module). This is standard if $A$ is unital, but in the general case one can just adjoin a unit to $A$ and note that modules over $A$ are in bijective correspondence with unital modules over $A^{\sim}$. Next, recall that by Theorem 26 of [13], $e$ is similar in $\mathrm{M}_{n}\left(A^{\sim}\right)$ to a self-adjoint idempotent (i.e., projection), and so we may suppose that $e$ is self-adjoint. Finally, note that, presumably, any two Hilbert $A$-modules which are isomorphic as $A$-modules are in fact isomorphic as Hilbert $A$-modules, but, this is quite elementary in the case that one of them is $e\left(A^{\sim} \oplus \cdots \oplus A^{\sim}\right)$ as above. Namely, any module map from $e\left(A^{\sim} \oplus \cdots \oplus A^{\sim}\right)$ to a Hilbert $A^{\sim}$-module (or even just to a Banach $A$-module) $X$ must be continuous, since this is trivially true in the case $e=1$-and just $A^{\sim}$ in place of $A^{\sim} \oplus \cdots \oplus A^{\sim}$. In fact, it must be compact, since the identity of $e\left(A^{\sim} \oplus \cdots \oplus A^{\sim}\right)$ is compact. (Since this is even finite rank, one sees that the composed map is also of finite rank - and so we do not have to prove it is continuous.) Then just pass to the unitary part of the polar decomposition of this continous invertible map - to obtain an isomorphism of Hilbert $A^{\sim}$-modules, from the existence of which one concludes that $e\left(A^{\sim} \oplus \cdots \oplus A^{\sim}\right)$ is in fact also a Hilbert A-module (i.e., the $A^{\sim}$-valued inner product takes values in $A$ ). (That we are in the setting of elementary $\mathrm{C}^{*}$-algebra theory may be seen by noting that the map belongs to the compact endomorphism $\mathrm{C}^{*}$-algebra of the direct sum of the two modules.)

Conversely, let $X$ be a countably generated Hilbert $A$-module which, in the Cuntz semigroup, is order-theoretically compact. Let us show that the $\mathrm{C}^{*}$-algebra of compact endomorphisms of $X$ is unital. If not, since it has a strictly positive element (see above), it has an increasing approximate unit $0 \leq u_{1} \leq u_{2} \leq \cdots$ such that $u_{i+1} u_{i}=u_{i}$ and $u_{i+1} \neq u_{i}$ for every $i$. As shown earlier,

$$
[X]=\sup \left[\left(u_{i} X\right)^{-}\right],
$$


and here by compactness, $[X] \leq\left[\left(u_{i} X\right)^{-}\right]$for some $i$. By Theorem 3 , as $A$ has stable rank one $X$ is isomorphic to a subobject of $\left(u_{i} X\right)^{-}$. It follows that if $X \neq\left(u_{i} X\right)^{-}$ then the $\mathrm{C}^{*}$-algebra $B$ of compact endomorphisms of $X$ has a scaling element in the sense of $[2]$.

(If $b$ is a self-adjoint element of $B$ equal to the identity on $X_{i}=\left(u_{i} X\right)^{-}$, and $v$ is an isomorphism from $X$ to a subobject of $X_{i}$, then $v b \in B$ (indeed, to see this it is enough to consider the case $b$ has finite rank, say $b$ is the map $\xi \mapsto \eta\langle\zeta, \xi\rangle$ for some $\eta, \zeta \in X$, and then $v b$ is the map $\xi \mapsto v \eta\langle\zeta, \xi\rangle)$. With $x=v b$, we have $x^{*} x=b v^{*} v b=b^{2}$ and $x x^{*}=v b^{2} v^{*}$, and so $\left(x^{*} x\right)\left(x x^{*}\right)=b^{2} v b^{2} v^{*}=v b^{2} v^{*}=x x^{*}$. Furthermore, if $x^{*} x=x x^{*}$ then $b^{4}=b^{2}$, i.e., $b^{2}$ is a projection, and, furthermore, $v b^{2} v^{*}=b^{2}$, which implies that $b^{2}$ is exactly the unit of the compact endomorphism algebra of $X_{i}$, which is impossible by hypothesis. Thus, $x^{*} x \neq x x^{*}$, and so $x$ is a scaling element.)

Hence, by the proof of Theorem 3.1 of [2], the $\mathrm{C}^{*}$-algebra $A^{\sim}$ obtained by adjoining a unit to $A$ has an infinite projection. This contravenes the hypothesis that $A$-or rather (by definition), $A^{\sim}$ - has stable rank one (used now for the second time in the proof of this implication). (To show that a unital $\mathrm{C}^{*}$-algebra of stable rank one does not have an infinite projection, it is enough to show that the unit cannot be infinite, or, equivalently, that every isometry is unitary. But if an isometry can be approximated arbitrarily closely by an invertible element, then it can also be approximated arbitrarily closely by the unitary part of this invertible element, and so its range projection also can be approximated arbitrarily closely by the unit, and so must be the unit; the isometry must be unitary.)

Now consider the second assertion of the theorem. Suppose first that $A$ has real rank zero, and let us show that every element of $\mathcal{C} u(A)$ is the supremum of an increasing sequence of compact elements. Let $X$ be a countably generated Hilbert $A$-module. Since by Theorem 2 of [14], $X$ is isomorphic to a closed submodule of $A \oplus A \oplus \cdots$ (cf. just above), the $\mathrm{C}^{*}$-algebra of compact endomorphisms of $X$ is isomorphic to a hereditary sub- $C^{*}$-algebra of the stabilization of $A$ and is therefore also of real rank zero. In particular - since it has a countable approximate unit (see proof of Theorem 1) - this $C^{*}$-algebra has an increasing sequential approximate unit $\left(e_{n}\right)$ consisting of projections. Then $X=\lim _{\rightarrow} e_{n} X$, and so by the proof of Theorem $1,[X]=\sup \left[e_{n} X\right]$ in $\mathcal{C} u(A)$. As shown above, $\left[e_{n} X\right]$ is compact in $\mathcal{C} u(A)$ for each $n$. (The proof of this first implication does not use that $A$ is simple or has stable rank one.)

Now suppose that every element of $\mathcal{C} u(A)$ is the supremum of an increasing se- 
quence of compact elements, and let us show that $A$ has real rank zero. By Theorem 2.6 of [3], it suffices to show that any singly generated hereditary sub- ${ }^{*}$-algebra of $A$, say $B$, has an increasing approximate unit consisting of projections, or, equivalently, that the closed right ideal $B$ generates, say $X$, is the closure of an increasing sequence of closed right ideals generated by projections,- - or, equivalently again, that $X$ is the Hilbert $A$-module inductive limit of a sequence of closed right ideals of $A$ generated by projections, with respect to Hilbert $A$-module isomorphisms between the objects at various stages and subobjects of the succeeding ones. (This is clearly equivalent to the same statement for the increasing sequence of images of these objects in $X$, and any subobject of $X$ is a closed right ideal, necessarily generated by a projection if this is true up to isomorphism, by the first assertion of the theorem.)

By hypothesis, $[X]=\sup x_{i}$ in $\mathcal{C} u(A)$ where $x_{1} \leq x_{2} \cdots$ is an increasing sequence of compact elements of $\mathcal{C} u(A)$. With $X_{1}, X_{2} \cdots$ Hilbert $\mathrm{C}^{*}$-modules such that $x_{i}=\left[X_{i}\right]$ for each $i$, by Theorem 3 (as $A$ has stable rank one) $X_{i}$ is isomorphic to a subobject of $X_{i+1}$ for each $i$. As shown in the proof of Theorem 1, in this case

$$
\sup \left[X_{i}\right]=\left[\lim _{\rightarrow} X_{i}\right]
$$

By Theorem 3 again (actually, the first use of Theorem 3 above was not necessary on account of the very special nature of the Hilbert $A$-modules $X_{i}$-algebraically finitely generated and projective by the first assertion of the theorem),

$$
X \cong \lim _{\rightarrow} X_{i}
$$

where $\cong$ denotes isomorphism of Hilbert $A$-modules. In particular, we may suppose that each $X_{i}$ is a subobject of $X$ and that the maps $X_{i} \rightarrow X_{i+1}$ are inclusions. Since $X$ is a closed right ideal of $A$ it follows that $X_{i}$ is also for each $i$, and it remains to note that also $X_{i}=e_{i} A$ for a projection $e_{i} \in A$. (Otherwise $X_{i}$ could not be finitely generated algebraically, let alone be projective!)

6. Appendix. Let us explain in more detail the relationship between the ordered semigroup $\mathcal{C} u A$ defined in Section 1 (and studied in Theorems 1, 2, and 3 and Corollaries 4 and 5 above) and the ordered semigroup introduced by Cuntz in [4]denoted by W $A$ by Rørdam in [25] and now often called the Cuntz semigroup. (In [25] the structure of WA was referred to as that of positive ordered semigroup, to reflect the fact that every element is positive. In the present article we suppress this additional qualifier, since we are only considering such ordered abelian semigroups.) 
(The main purpose of our proposed new notation is to emphasize the additional structure we have identified - the operation of taking countable increasing suprema and the relation of compact containment in the order-theoretic sense.)

Briefly, if $A$ is stable, i.e., if $A$ is isomorphic to $A \otimes \mathfrak{K}$ where $\mathfrak{K}$ denotes the $\mathrm{C}^{*}$-algebra of compact operators on a separable infinite-dimensional Hilbert space, then the two ordered semigroups are exactly the same (the functors are equivalent by a natural transformation).

In general, $\mathcal{C} u A$ is the same as $\mathcal{C} u(A \otimes \mathfrak{K})$ (and so the same as $\mathrm{W}(A \otimes \mathfrak{K}))$

Let us show first that, with $\mathcal{C} u A$ defined as in Section $1, \mathcal{C} u A$ is isomorphic to $\mathcal{C} u(A \otimes \mathfrak{K})$ - and that the isomorphism may be chosen to be natural (i.e., to be a natural transformation between these two functors).

By the functoriality of $\mathcal{C} u$ (Theorem 2 above), corresponding to the inclusion of $A$ as $A \otimes e$ in $A \otimes \mathfrak{K}$, where $e$ is a (fixed) minimal non-zero projection in $\mathfrak{K}$, there is a morphism $\mathcal{C} u A \rightarrow \mathcal{C} u(A \otimes \mathfrak{K})$ in the category $\mathcal{C} u$, which furthermore (again by functoriality) constitutes a natural transformation between these two functors. It remains to show that this map is an isomorphism for any $\mathrm{C}^{*}$-algebra $A$.

On specializing to the present case, the morphism $\mathcal{C} u A \rightarrow \mathcal{C} u(A \otimes \mathfrak{K})$ is seen to consist of, given a countably generated (right) Hilbert $A$-module $X$, taking the Hilbert $A$-module direct sum of a countable infinity of copies of $X$, and then having both $A$ and $\mathfrak{K}$-and therefore also $A \otimes \mathfrak{K}$-act on this in the natural way (on the right). Let us show that the map in the opposite direction, beginning with a countably generated Hilbert $A \otimes \mathfrak{K}$-module $Y$, and cutting it down by the subalgebra $A \otimes e$ where $e$ is a fixed minimal non-zero projection in $\mathfrak{K}$, resulting in a Hilbert $\mathrm{C}^{*}$-module over this $\mathrm{C}^{*}$-algebra which is naturally isomorphic to $A$, and which is countably generated if $Y$ is, preserves our notion of equivalence of countably generated Hilbert $\mathrm{C}^{*}$-modules, and at the level of Cuntz semigroups (in the sense of the present paper) is the inverse of the map just described. To see that equivalence is preserved by the backwards map, it is enough to note that this map preserves isomorphism (it in fact preserves arbitrary homomorphisms in a natural way), and both this map and the map in the forwards direction preserves the relation of inclusion and the relation of compact containment (for a subobject - see Theorem 1). It is straightforward that these maps are inverse to each other at the level of Hilbert $\mathrm{C}^{*}$-modules, and the desired isomorphism of the Cuntz semigroups follows.

Let us now show that, if $A$ is stable, then the map which to a positive element of $A$ associates the closed right ideal it generates, considered as a Hilbert $A$-module, determines an isomorphism between $\mathrm{W} A$ and $\mathcal{C} u A$.

Recall that two positive elements of $A$, let us say $a$ and $b$, are comparable in the 
sense of Cuntz, with $b$ majorizing $a$, if there exists a sequence $\left(c_{n}\right)$ in $A$ such that $c_{n} b c_{n}^{*}$ converges to $a$. Let us suppose that this holds, and let us show that the class in $\mathcal{C} u A$ of the closure of $a A$ is majorized by the class of the closure of $b A$.

By Lemma 2.2 of [17], for each $n$ there exists $d_{n}$ in $A$ such that $d_{n} c_{n} b c_{n}^{*} d_{n}^{*}$ is a continuous function $a_{n}$ of $a$ and the sequence $\left(a_{n}\right)$ is increasing with limit $a$. It follows on the one hand that the closure of $a_{n} A$ is isomorphic to a subobject of the closure of $b A$ for each $n$, and in particular the class of this Hilbert $A$-module in $\mathcal{C} u A$ is majorized by the class of the closure of $b A$, and on the other hand, as shown in the proof of Theorem 1 , that the class of the closure of $a A$ in $\mathcal{C} u A$ is the supremum of the increasing sequence of classes of the closures of $a_{1} A, a_{1} A, \cdots$. It follows immediately that the class of the closure of $a A$ in $\mathcal{C} u A$ is majorized by the class of the closure of $b A$.

Let us show, conversely, that if $a$ and $b$ are as above, and the closed right ideal of $A$ generated by $a$ is majorized in the ordered semigroup $\mathcal{C} u A$ defined above (actually proved to be an ordered semigroup only in the proof of Theorem 1) by the closed right ideal generated by $b$, then $a$ is majorized by $b$ in the sense of Cuntz. Choose a continuous positive real-valued function $f$ on the spectrum of $a$ equal to zero in a neighbourhood of zero (if zero belongs to the spectrum of $a$ ), such that $f(a)$ is close to $a$. Then the closed right ideal generated by $f(a)$ is compactly contained in that generated by $a$ (as there exists a continuous function $g$ on the spectrum of $a$, equal to zero at zero, such that $g(a) f(a)=f(a))$, and is therefore by hypothesis isomorphic, as a Hilbert $A$-module, to a subobject (compactly contained, but we shall not need this) of the closed right ideal generated by $b$. Such a subobject must in fact be a smaller closed right ideal, countably generated and therefore singly generated. It follows that there is an element $x$ of $A$ such that $x^{*} x=f(a)$ and $x x^{*}$ generates the closed right ideal in question. Since then $x$ is also in this ideal, which is contained in the closed right ideal generated by $b$, there is a sequence $\left(a_{n}\right)$ in $A$ such that $b a_{n}$ converges to $x$, and then $f(a)$ is the limit of $x^{*} b a_{n}$. By polarization (namely, the equation $c b d^{*}$ equal to the average of the elements $(c+z d) b(c+z d)^{*}$ with $z$ a power of $i$, which holds as $b$ is self-adjoint), $x^{*} b a_{n}$ is majorized by $b$ in the sense of Cuntz for each $n$, and hence, since the set of such elements is closed (as follows immediately from the definition), also $f(a)$ is majorized by $b$ in this sense - indeed, since $f(a)$ is arbitrarily close to $a$, also $a$ is.

It follows that the map from positive elements of $A$ to closed right ideals gives rise to an isomorphism of ordered semigroups between $\mathrm{W} A$ and $\mathcal{C} u A$, or, rather, in the first instance, between the subset of W $A$ arising from positive elements of $A$, as opposed to matrix algebras over $A$, and the subset of $\mathcal{C} u A$ arising from singly 
generated closed right ideals of $A$, as opposed to countably generated Hilbert $\mathrm{C}^{*}$ modules over $A$-but these subsets exhaust these two semigroups in the case that $A$ is stable, as we shall now show.

First, let a be a positive element of $A \otimes \mathrm{M}_{n}$, and let us show that it is equivalent in $\mathrm{W} A$ to a positive element of $A \otimes e$, where $e$ is a non-zero minimal projection in $\mathrm{M}_{n}$, using of course that $A$ is stable. Writing $A$ as $B \otimes \mathfrak{K}$ for some $B$, and noting that there is an isometry $v$ in the multiplier $C^{*}$-algebra of $\mathfrak{K} \otimes \mathrm{M}_{n}$ such that $v\left(\mathfrak{K} \otimes \mathrm{M}_{n}\right) v^{*}=\mathfrak{K} \otimes e$, we have that $(1 \otimes v) a(1 \otimes v)^{*}$ is a positive element of $\mathfrak{K} \otimes e$, which is easily seen to be equivalent in WA to $a$. (Recall that $v$ is the limit of a sequence of elements of $\mathfrak{K} \otimes \mathrm{M}_{n}$ ) in the strict topology on the multiplier algebra.)

Second, and finally, let $X$ be a countably generated Hilbert $\mathrm{C}^{*}$-module over $A$, and let us show that $X$ is isomorphic to a singly generated closed right ideal of $A$ (using again that $A$ is stable). It is enough to show that, as a Hilbert $A$-module, $X$ is isomorphic to just some closed right ideal of $A$, since this is then countably generated as $X$ is, and a countably generated closed right ideal of a $\mathrm{C}^{*}$-algebra is singly generated. (A countable set of generators may be assumed to be positive and summable in norm, and the sum is then a single generator - as the self-adjoint part of a closed two-sided ideal is a hereditary sub- $\mathrm{C}^{*}$-algebra.)

By Theorem 2 of [14], $X$ is isomorphic to a direct summand of the Hilbert $\mathrm{C}^{*}$ module direct sum of a countable infinity of copies of the Hilbert $A$-module $A$, and in particular to a closed submodule of this direct sum. In fact, since $A$ is stable, this infinite direct sum Hilbert $A$-module is isomorphic to $A$ !

(To see this, write $A$ as $B \otimes \mathfrak{K}$ for some $\mathrm{C}^{*}$-algebra $B$, and note that the Hilbert $A$-module $A$ is equal to the (internal) Hilbert $\mathrm{C}^{*}$-module direct sum of the closed right ideals $\left(1 \otimes e_{n}\right)(B \otimes \mathfrak{K})$ where $\left(e_{n}\right)$ is a sequence of minimal closed two-sided projections in $\mathfrak{K}$ with sum equal to one in the multiplier $\mathrm{C}^{*}$-algebra of $\mathfrak{K}$. In other words, $A$ is isomorphic as a Hilbert $A$-module to an infinite direct sum of copies of some Hilbert $A$-module, and partitioning the index set into a countable infinity of subsets of the same cardinality as the whole set one sees that $A$ is isomorphic to a countably infinite direct sum of copies of itself.)

It follows that $X$ is isomorphic to a closed right ideal of $A$, and a countably generated one since $X$ is countably generated. Recall, finally, that a countably generated closed right ideal of a $\mathrm{C}^{*}$-algebra is singly generated.

One advantage of the original description of the Cuntz semigroup of a $\mathrm{C}^{*}$-algebra is that it is immediate that approximately inner automorphisms of the $\mathrm{C}^{*}$-algebra act trivially on it. (In the Hilbert $\mathrm{C}^{*}$-module setting, which is remarkably useful for a number of purposes, as may be clear by now-for instance, in the case of a 
$\mathrm{C}^{*}$-algebra of stable rank one Cuntz equivalence just amounts to isomorphism of Hilbert modules, in perfect analogy with Murray-von Neumann equivalence - it is perhaps not quite obvious that even inner automorphisms act trivially. In fact, a straightforward algebraic calculation establishes this.)

Another advantage of the original description of the Cuntz semigroup is that, for a non-stable $\mathrm{C}^{*}$-algebra, it contains additional information: just as in the case of the Murray-von Neumann semigroup, one may keep track of when additional classes appear when one passes to matrix algebras - or, for that matter, when one stabilizes (although in the case of the Murray-von Neumann semigroup this last step introduces no new classes). On the other hand, this information is also readily expressible in the Hilbert module language. Just as projections in a matrix algebra of a certain order over an arbitrary algebra correspond to projective modules having a generating subset with that number of elements, so also do Cuntz classes arising in the original sense from a matrix algebra of a certain order over a $\mathrm{C}^{*}$-algebra correspond to Hilbert $\mathrm{C}^{*}$-modules over the $\mathrm{C}^{*}$-algebra with that (finite) number of generators - it is only when one looks at Cuntz classes arising from the stabilization that one obtains a Hilbert $\mathrm{C}^{*}$-module requiring an infinite number of generators. (On the other hand, for most purposes it is already of interest to consider the case of a stable $\mathrm{C}^{*}$-algebra, in which case every countably generated Hilbert $\mathrm{C}^{*}$-module is singly generated, as a Hilbert $\mathrm{C}^{*}$-module.) (Note that a Hilbert $\mathrm{C}^{*}$-module which is countably generated purely algebraically, i.e., as a module, must in fact be finitely generated and projective, as a module.)

\section{References}

1. B. Blackadar, K-Theory for Operator Algebras (Second edition), Mathematical Sciences Research Institute Publications, 5, Cambridge University Press, Cambridge, 1998.

2. B. Blackadar and J. Cuntz, The structure of stable algebraically simple $C^{*}$ algebras, Amer. J. Math. 104 (1982), 813-822.

3. L. G. Brown and G. K. Pedersen, $C^{*}$-algebras of real rank zero, J. Funct. Anal. 99 (1982), 131-149.

4. J. Cuntz, Dimension functions on simple $C^{*}$-algebras, Math. Ann. 233 (1978), 145-153.

5. G. A. Elliott, The ideal structure of the multiplier algebra of an AF algebra, C. R. Math. Acad. Sci. Soc. R. Can. 9 (1987), 225-230. 
6. G. A. Elliott, On the classification of $C^{*}$-algebras of real rank zero, J. Reine Angew. Math 443 (1993), 179-219.

7. G. A. Elliott, Towards a theory of classification, preprint.

8. G. A. Elliott, G. Gong, and L. Li, On the classification of simple inductive limit $C^{*}$-algebras II: The isomorphism theorem, preprint, 1998 (to appear, Invent. Math.).

9. G. A. Elliott and C. Ivanescu, The classification of separable simple $C^{*}$ algebras which are inductive limits of continuous-trace $C^{*}$-algebras with spectrum homeomorphic to the closed interval [0,1], preprint.

10. G. A. Elliott and K. Kawamura, A Hilbert bundle characterization of Hilbert $C^{*}$-modules, preprint.

11. T. M. Ho, On inductive limits of homogeneous $C^{*}$-algebras with diagonal morphisms between the building blocks, Ph.D. thesis, University of Toronto, 2006.

12. K. K. Jensen and K. Thomsen, Elements of KK-Theory, Mathematics: Theory and Applications, Birkhäuser, Basel, 1991.

13. I. Kaplansky, Rings of Operators, W. A. Benjamin, Inc., New York, 1968.

14. G. G. Kasparov, Hilbert $C^{*}$-modules: theorems of Stinespring and Voiculescu, J. Operator Theory 4 (1980), 133-150.

15. E. C. Lance, Hilbert $C^{*}$-modules. A tool kit for operator algebraists, London Mathematical Society Lecture Note Series, 210, Cambridge University Press, Cambridge, 1995.

16. E. Kirchberg and M. Rørdam, Non-simple purely infinite $C^{*}$-algebras, Amer. J. Math. 122 (2000), 637-666.

17. E. Kirchberg and M. Rørdam, Infinite non-simple $C^{*}$-algebras absorbing the Cuntz algebra $\mathcal{O}_{\infty}$, Adv. Math. 167 (2002), 195-264.

18. H. Lin, An introduction to the classification of amenable $C^{*}$-algebras, World Scientific Publishing Co., Inc., River Edge, NJ, 2001.

19. H. Lin, Simple AH algebras of real rank zero, Proc. Amer. Math. Soc. 131 (2003), 3813-3819.

20. H. Lin, Classification of simple $C^{*}$-algebras of tracial topological rank zero, Duke Math. J. 125 (2004), 91-119.

21. V. M. Manuilov and E. V. Troitsky, Hilbert $C^{*}$-modules. Translated from the 2001 Russian original by the authors. Translations of Mathematical Monographs, 226, American Mathematical Society, Providence, RI, 2005. 
22. M. Rørdam, On the structure of simple $C^{*}$-algebras tensored with a UHF algebra, II, J. Funct. Anal. 107 (1992), 255-269.

23. M. Rørdam, A short proof of Elliott's theorem: $\mathcal{O}_{2} \otimes \mathcal{O}_{2} \cong \mathcal{O}_{2}$, C. R. Math. Acad. Sci. Soc. R. Can. 16 (1994), 31-36.

24. M. Rørdam, Classification of nuclear, simple $C^{*}$-algebras, pages 1-145 of Classification of nuclear $C^{*}$-algebras. Entropy in operator algebras. Encyclopedia of the Mathematical Sciences, 126, Springer, Berlin, 2002.

25. M. Rørdam, The stable and the real rank of $\mathcal{Z}$-absorbing $C^{*}$-algebras, International J. Math. 15 (2004), 1065-1084.

26. A. S. Toms, On the classification problem for nuclear $C^{*}$-algebras, Ann. of Math., to appear.

Department of Mathematics, University of Toronto, Toronto, Ontario, Canada M5S 2E4 kris@math.toronto.edu elliott@math.toronto.edu

Department of Mathematics, Northern British Columbia, Prince George, British Columbia, Canada V2N 4Z9

cristian@math.toronto.edu 